\title{
Large-eddy simulation of a neutrally stratified boundary layer: A comparison of four computer codes
}

\author{
By A. ANDREN ${ }^{1 *}$, A. R. BROWN ${ }^{2}$, J. GRAF GR. J. MASON $^{2}$, C.-H. MOENG ${ }^{4}$, F. T. M. NIEUWSTADT \\ and U. SCHUMANN ${ }^{3}$ \\ ${ }^{1}$ Uppsala University, Sweden \\ ${ }^{2}$ Meteorological Office, $U K$ \\ ${ }^{3}$ DLR Institute of Atmospheric Physics, Germany \\ ${ }^{4}$ National Center for Atmospheric Research, USA \\ ${ }^{5}$ Delft University of Technology, The Netherlands
}

(Received 13 December 1993; revised 8 March 1994)

\begin{abstract}
SUMMARY
A neutrally stratified Ekman layer limited in depth by a stress-free rigid lid has been simulated using four different large-eddy simulation computer codes. This is the second large-eddy simulation intercomparison study in a series of simulations of different flows aimed at a systematic analysis of large-eddy simulation sensitivity to subgrid scale model assumptions and numerical methods. The resolution was chosen to be coarse so that the results might be sensitive to the details of the various subgrid scale model formulations. Stochastic backscatter of subgrid kinetic energy and scalar variance was included in one subgrid scale model.

Profiles of first and second moments, budgets of resolved-scale second moments, and spectra have been analyzed. It is found that significant differences in the lowest third of the boundary layer result from moderate changes in subgrid scale eddy diffusivities or from the inclusion of backscatter.

To isolate effects of the various numerical methods, a set of tests with a fixed subgrid scale K-profile were made. When run with a common subgrid scale model the results from the four large-eddy simulation codes converge. This test indicates that the large-eddy simulation results are more sensitive to the subgrid scale model formulations than to the various numerics.
\end{abstract}

\section{INTRODUCTION}

Large-eddy simulation (LES) has become a useful tool to investigate characteristics of turbulent flows. Using this method, turbulent flow structures, transport dynamics, ensemble average statistics, and closure assumptions used in ensemble average modelling, have been examined within various types of planetary boundary layers (PBLs) (Nieuwstadt and Brost 1986; Mason 1989; Moeng and Wyngaard 1989; Schmidt and Schumann 1989; Andrén and Moeng 1993).

For fine grids, LES should be insensitive to the particular choice of subgrid scale (SGS) parametrization, except close to the ground where the turbulence scales must become smaller and an increased sensitivity to SGS parametrization is inevitable. However, results in the bulk of the boundary layer may be more sensitive to the subgrid model when using coarse grids which only marginally resolve the inertial subrange. In addition numerical details may also affect the simulation results.

In this paper, we present results from simulations of a particular flow by a set of LES codes that use a variety of SGS parametrizations as well as different numerical methods. The flow studied is a neutrally stratified atmospheric boundary layer limited in depth by a stress-free rigid lid, i.e. an analogue to the classical Ekman layer. A similar exercise was carried out by Nieuwstadt et al. (1992) (hereafter NMMS92) for a convective atmospheric boundary layer, using the same four LES codes as in the present study. NMMS92 found that all four LES simulations performed about equally well, i.e. differences, which typically were not greater than about $10 \%$, were found to lie within the scatter of available observations, even with a rather coarse resolution. The main differences between the various LES results were attributed to the use of different values of the so-called Smagorinsky constant in the SGS model, which is the ratio between the

* Corresponding author: Dept. Meteorology, Uppsala University, Box 516, S-751 20 Uppsala, Sweden. 
subgrid mixing length and a representative grid size. However, it was felt that the good agreement of all LES results was partly due to the fact that the convective boundary layer is a rather easy case for LES, because its structure is dominated by large-scale thermals. It was therefore decided to extend the comparison to study a more challenging case, namely the neutral shear-driven boundary layer. In this type of boundary layer the turbulent eddies are smaller than in the convective boundary layer case, and the inertial subrange is reached at higher wavenumbers. For given domain size and grid resolution a larger impact of the SGS formulation is thus expected in a neutral case than in a convective case. This makes the neutral boundary layer more difficult to simulate by the LES technique since one has to rely more on the SGS model.

We consider a neutral turbulent Ekman layer, i.e. a flow driven by a constant large scale pressure gradient in a rotating system. Resolved-scale turbulent motions are thus due to shear instabilities. A passive scalar variable is introduced into the simulations by imposing a constant flux at the surface. All LESs were started from the same initial conditions and used the same forcing and boundary conditions.

First, in section 2, we discuss the LES codes with emphasis on differences, specifically SGS formulations and numerical methods. Next, in section 3 , we describe the calculation set up and the boundary conditions. The LES results are presented in section 4 . In section 5 we discuss results from a test in which all LES codes were run with a common SGS model, and finally conclusions are given in section 6 .

\section{OVERVIEW OF THE LES CODES}

We will refer to the four different LES computer codes (and the co-authors undertaking the investigations) as Mason's (Mason-Brown), Moeng's (Andren-Moeng), Nieuwstadt's (Nieuwstadt), and Schumann's (Schumann-Graf). Detailed descriptions of the individual codes may be found in Mason (1989); Moeng (1984); Nieuwstadt and Brost (1986) and Schmidt and Schumann (1989), respectively. The backscatter model used in Mason's code is described in Mason and Thomson (1992). Here we will focus on the differences in the models' SGS formulations and numerical techniques.

\section{(a) Subgrid scale models}

By filtering the Navier-Stokes equations, turbulent motions can be separated into the resolved and subgrid scales. The conservation equations for resolved-scale velocities and scalars for incompressible flow may be written as

and

$$
\frac{\partial \bar{u}_{i}}{\partial t}=-\frac{\partial \bar{u}_{j} \bar{u}_{i}}{\partial x_{j}}+f U_{\mathrm{g}} \delta_{i 1}-f V_{\mathrm{g}} \delta_{i 2}-\frac{\partial}{\partial x_{i}}\left(\frac{\bar{p}_{\mathrm{r}}}{\rho}+\frac{2 e}{3}\right)-\frac{\partial \tau_{i j}}{\partial x_{j}}-2 \varepsilon_{i j k} \Omega_{j} \bar{u}_{k},
$$

$$
\frac{\partial \bar{c}}{\partial t}=-\frac{\partial \bar{u}_{i} \bar{c}}{\partial x_{i}}-\frac{\partial \tau_{c i}}{\partial x_{i}},
$$

where an overbar denotes a filtered variable, $U_{\mathrm{g}}$ and $V_{\mathrm{g}}$ are the geostrophic wind components, $f$ is the vertical Coriolis component, $\bar{p}_{\mathrm{r}}$ is the resolved-scale pressure field, $\rho$ is the (constant) density, $e$ is the subgrid turbulent kinetic energy (TKE), $\tau_{i j}$ and $\tau_{c i}$ are the anisotropic parts of the SGS stresses, and $\Omega_{j}$ is the earth rotation vector. Other forms of these equations exist and e.g. Moeng's code uses a vorticity form of Eq. (1). Note that we have defined TKE in this incompressible flow to be equal to half the sum of the three velocity variances $\left(\mathrm{m}^{2} \mathrm{~s}^{-2}\right)$.

Whereas the resolved scales are explicitly calculated, SGS motions must be parametrized. Until quite recently, it was assumed that the only role of the SGS model is 
to dissipate the energy that is passed down to small scales through the energy cascade process. Typically this was achieved by using the Smagorinsky (1963) formulation for the anisotropic part of the SGS stresses

$$
\tau_{i j}=-K_{\mathrm{M}}\left(\frac{\partial \bar{u}_{i}}{\partial x_{j}}+\frac{\partial \bar{u}_{j}}{\partial x_{i}}\right),
$$

where the eddy viscosity, $K_{M}$, is given as $R^{2} S$. Here $S=\sqrt{\frac{1}{2} S_{i j}^{2}}=\sqrt{\frac{1}{2}\left(\partial \bar{u}_{i} / \partial x_{j}+\partial \bar{u}_{j} / \partial x_{i}\right)^{2}}$ is the absolute value of the strain rate of the resolved-scale velocity field and $l$ is a representative SGS length scale. In Smagorinsky's original formulation $l=C_{\mathrm{s}} \Delta$, where $\Delta$ is a characteristic grid size. Mason's code, without the option of backscatter of kinetic energy and scalar variance (see below), uses this formulation.

In both Moeng's and Nieuwstadt's codes, $K_{\mathrm{M}}$ is given by

$$
K_{\mathrm{M}}=C L e^{1 / 2} \text {, }
$$

where the subgrid TKE is calculated from a prognostic equation and where $L$ is a representative subgrid length scale. Lastly, in Schumann's code, the SGS formulation is also based on a prognostic equation for $e$. However, Schumann's $K_{\mathrm{M}}$ formula is based on a single-point closure scheme for the second moments of the subgrid fluctuations. Nevertheless, in the present neutral flow simulation, Schumann's SGS formulation differs from that in Moeng's and Nieuwstadt's codes only by the values of SGS closure constants for diffusion and dissipation. In these simulations Schumann's code does not include the splitting of diffusivities in a locally 'isotropic' and 'homogeneous' part as in Schumann (1975). In the codes of Moeng, Nieuwstadt and Schumann, the SGS length scale $L$ in the interior of the flow is proportional to $\Delta$. However, the codes use different definitions of $\Delta$.

To clarify differences in the SGS formulations we assume that shear production is balanced with dissipation in the SGS TKE budgets. With this balance we obtain the anisotropic SGS stresses as $\tau_{i j}=-\mathcal{R} S S_{i j}=-K_{\mathrm{M}} S_{i j}$, as in Eq. (3), and may calculate $l=$ $\left(K_{\mathrm{M}} / S\right)^{1 / 2}$ for the three codes. These values can be compared with the value of $l$ used by Mason, which is prescribed directly without any explicit reference to $\Delta$. Finally we can extract the values of $C_{\mathrm{s}}$ using the common definition $l=C_{\mathrm{s}}(\Delta x \Delta y \Delta z)^{1 / 3}$.

The SGS length scale is constant in Moeng's code. Close to the surface, Mason's and Schumann's codes set the SGS length scale to be proportional to height, while Nieuwstadt's code effectively attains this proportionality by using a different formulation for the dissipation term in the equation for subgrid TKE near the surface. All the codes relate the SGS eddy diffusivity for scalars, $K_{C}$, to the SGS eddy diffusivity for momentum, $K_{\mathrm{M}}$, through a constant SGS Schmidt number, $S c$,

$$
S c=\frac{K_{\mathrm{M}}}{K_{\mathrm{C}}}
$$

Values of SGS Schmidt number, $l$ and $C_{\mathrm{s}}$ used are given in Table 1.

A longstanding problem in LES of neutrally stratified, high Reynolds number flows has been an inability to obtain a realistic logarithmic velocity profile in the surface layer, with excessive shear almost invariably found in the semi-resolved region close to the surface. The Smagorinsky model is purely dissipative and is deterministic as it assumes that subgrid motions are fully determined by the resolved motions. However, it has been recognized (e.g. Leslie and Quarini 1979) that SGS stresses should have stochastic fluctuations, and that the effect of these fluctuations is to 'backscatter' some energy from small to large scales, against the mean direction of energy transfer along the cascade. 
Mason and Thomson (1992) used a stochastic subgrid forcing model in which the rates of backscatter of TKE and scalar variance were $C_{\mathrm{B}}$ times the dissipation of energy, and $C_{\mathrm{BC}}$ times the rate of dissipation of scalar variance, respectively. The magnitude of these constants can be estimated from the EDQNM (Eddy-Damping Quasi-Normal Markovian) theory, in which the equation for third moments is closed by assuming that fourth-order cumulants provide an eddy damping effect on triad interactions (Lesieur 1990), but, in view of the qualitative uncertainties, the values were empirically tuned to give the best results, i.e. logarithmic mean profiles. The spatial scale of fluctuations was dealt with by filtering the point wise random numbers with a three-dimensional $1: 2: 1$ filter and the temporal variation was treated crudely by varying the random numbers every two time steps. Nevertheless, using the backscatter SGS model, Mason and Thomson showed that the mean profiles had a marked improvement in the near-wall behaviour. In this study, Mason-Brown have performed simulations both with and without the backscatter effect. Values of $C_{\mathrm{B}}=1.40$ and $C_{\mathrm{BC}}=0.45$ are used in this study.

TABLE 1. SUMMARY OF INTERIOR VALUES OF $l$, SGS COEFFICIENTS $\left(C_{\mathrm{s}}\right)$, AND SGS SCHMIDT NUMBERS USED IN THE INDIVIDUAL LES CODES

\begin{tabular}{lccc}
\hline \multicolumn{1}{c}{ Code } & $l(\mathrm{~m})$ & $C_{\mathrm{s}}$ & $S c$ \\
\hline Mason's & 10.0 & 0.17 & 0.70 \\
Moeng's & 13.6 & 0.24 & 0.33 \\
Nieuwstadt's & 12.7 & 0.22 & 0.33 \\
Schumann's & 7.6 & 0.13 & 0.42 \\
\hline
\end{tabular}

\section{(b) Numerical solution techniques}

Uniform vertical grid-structures are employed in all codes except Mason's. In Mason's code the vertical mesh-spacing is reduced from about $40 \mathrm{~m}$ at the top of the domain to about $10 \mathrm{~m}$ as the surface is approached. To facilitate the present intercomparison, Mason-Brown have performed runs with a uniform vertical grid as well.

All codes use a vertically staggered grid. In addition, Nieuwstadt's, Mason's and Schumann's codes are staggered in the horizontal directions. Moeng's code uses the pseudo-spectral representation in the horizontal directions and is not horizontally staggered. The grid structure of each code is given in Fig. 1.

The various numerical schemes used are summarized below:

- Mason's and Nieuwstadt's codes:

Advection terms are solved by the Piacsek and Williams (1970) variance conserving second-order method, and time stepped by the leap-frog scheme with a weak time filter. Diffusion terms are second-order central differences and time advanced by the first-order forward Euler method.

- Moeng's code:

Advection terms are designed to conserve integrals of momentum and energy for the velocity fields, following Mansour et al. (1979), and volume integrated variances of the scalar fields. All horizontal derivatives are calculated by the pseudo-spectral method whereas vertical derivatives are approximated by second-order central differences. The second-order Adams-Bashforth time stepping is used for time integration.

- Schumann's code:

Advection of velocity components is approximated by second-order energy-conserving 
central differences, as first used by Lilly (1965). Advection of scalars and subgrid energy is done using the second-order upwind-scheme of Smolarkiewicz (1984), which guarantees positivity of scalar fields but causes some numerical diffusion of second order. Second-order central differences are used for the diffusion terms. The AdamsBashforth scheme is used for time advancement.

In order to reduce discretization errors and to weaken stability limits on the time step, a Galilean transformation is used by Mason-Brown, Nieuwstadt and SchumannGraf where the grid moves with velocities $\left(U_{\mathrm{Gal}}, V_{\mathrm{Gal}}\right)=(7.5,0.0) \mathrm{m} \mathrm{s}^{-1},\left(U_{\mathrm{Gal}}, V_{\mathrm{Gal}}\right)=$ $(10.0,0.0) \mathrm{m} \mathrm{s}^{-1}$ and $\left(U_{\mathrm{Gal}}, V_{\mathrm{Gal}}\right)=(7,2) \mathrm{m} \mathrm{s}^{-1}$ respectively.

The Piacsek and Williams (1970) advection scheme, used in Nieuwstadt's and Mason's codes, is equivalent to that of Lilly (1965), used in Schumann's code, when the continuity equation is satisfied.

All codes solve a Poisson equation for pressure through a mixed fast-Fourier and finite difference technique.

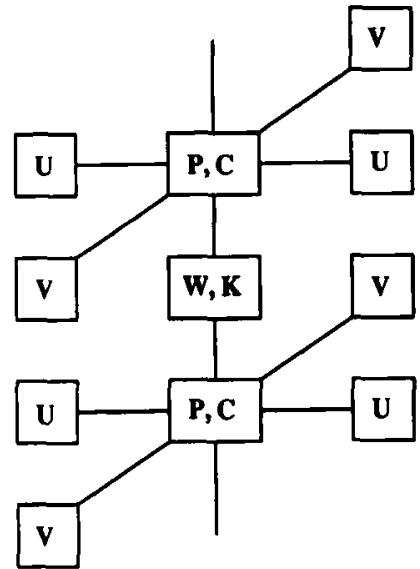

Mason's code

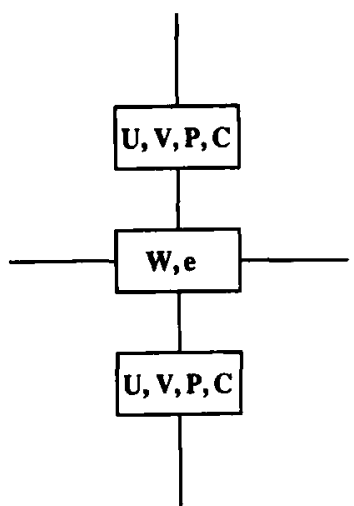

Moeng'scode

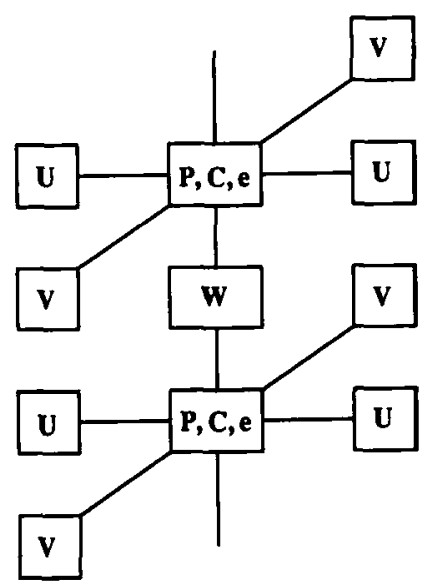

Nieuwstadt's and Schumann's codes

Figure 1. Schematic diagrams showing the grid structures used in the individual LES codes. 


\section{FLOW SET-UP AND BOUNDARY CONDITIONS}

We focus here on a PBL at the latitude of 45 degrees north, driven by a constant large-scale pressure gradient which, above the boundary layer, would balance a geostrophic wind of $\left(U_{\mathrm{g}}, V_{\mathrm{g}}\right)=(10.0,0.0) \mathrm{m} \mathrm{s}^{-1}$. We include in these simulations both vertical and horizontal components of the Coriolis force. The flow was simulated with a grid of $40 \times 40 \times 40$ points in a horizontally periodic box of $4000 \mathrm{~m} \times 2000 \mathrm{~m} \times 1500 \mathrm{~m}$ in $x$-, $y$ and $z$-directions, respectively. In view of the well known streak-like structures present near the wall in shear-driven boundary layers, a horizontally anisotropic grid was chosen in order to obtain a better spanwise resolution.

At the lower surface, we applied surface-layer similarity with a roughness length $z_{0}$ of $0.1 \mathrm{~m}$. A stress-free upper boundary was used. The height of this boundary is approximately $0.35 u_{*} / f$ in these runs, where $u_{*}=\left(\overline{u w}_{0}^{2}+\overline{v w}_{0}^{2}\right)^{1 / 4}$ is the surface friction velocity and the vertical component of the Coriolis parameter, $f$, is equal to $10^{-4} \mathrm{~s}^{-1}$. A passive scalar was introduced to the flow through a constant surface flux of $10^{-3} \mathrm{~kg} \mathrm{~m}^{-2}$ $\mathrm{s}^{-1}$. All codes use a density for air equal to $1 \mathrm{~kg} \mathrm{~m}^{-3}$.

The simulation was initialized by a steady state mean flow profile obtained from a PBL-model using a single-point closure turbulence scheme. To this mean flow, we added a small random perturbation in all velocity components, generated from random numbers uniformly distributed between -0.5 and 0.5 , with TKE $q^{2} / 2$, that is given in Table A.1 in the appendix. The simulations were run over a period of $10 \times f^{-1}$, of which the last $3 \times f^{-1}$ were used to calculate statistics for analysis. Statistics were built on equidistant samples from the analysis period but the number of samples varied among the codes as: Andren-Moeng, 18; Mason-Brown, 750; Nieuwstadt, 74; Schumann-Graf, 18.

Since a variety of numerical schemes as well as computer types have been employed in this study, it is of interest to summarize the CPU time usage in order to assess the computational efficiency of each individual code. Table 2 presents a summary of some key computational parameters. It should be noted that the CPU requirements are slightly unrepresentative since sections of the codes calculating buoyancy effects were retained although the effective force of gravity was identically zero.

\section{Results}

Including the four different runs made by Mason-Brown (i.e. non-uniform vertical grid and uniform vertical grid, both with and without backscatter), a total of seven different runs are available for comparison. However, compared with other variations, no significant differences were found between the Mason-Brown results with uniform and non-uniform grids, and all results presented are from their non-uniform grid runs except where otherwise stated. A comparison of results from essentially the same flow as simulated here, and, from observational data, has been made by Mason and Brown (1993). The scatter in the data does not allow critical discrimination between the results and in assessing the credibility of the simulations we have to place some reliance on the better known properties of surface layer flows and the expectation of a smooth match into the flow interior. We therefore limit ourselves to intercomparisons of the LES results from the different runs. In the presentation, we denote the horizontal average of a resolved-scale velocity component by, e.g., $\langle\bar{u}\rangle$. A second moment is denoted by, e.g., $\overline{u w}$, the net vertical turbulent u-momentum flux, which is a sum of the resolved-scale part $\left\langle\bar{u}^{\prime \prime} \bar{w}^{\prime \prime}\right\rangle=\langle(\bar{u}-\langle\bar{u}\rangle)(\bar{w}-\langle\bar{w}))\rangle$ and the SGS part, by $\left\langle\tau_{13}\right\rangle$. Results are normalized by the parameters, $u_{*}, f^{-1}$, and $c_{*}=\overline{w c}_{0} / u_{*}$. 
TABLE 2. SUMMARY OF COMPUTATIONAL STATISTICS

\begin{tabular}{|c|c|c|c|c|}
\hline Code & Mason's & Moeng's & Nieuwstadt's & Schumann's \\
\hline$N_{x}, N_{y}, N_{z}$ & 40 & 40 & 40 & 40 \\
\hline$L_{x}(\mathrm{~m})$ & 4000 & 4000 & 4000 & 4000 \\
\hline$L_{y}(\mathrm{~m})$ & 2000 & 2000 & 2000 & 2000 \\
\hline$L_{x}^{\prime}(\mathrm{m})$ & 1500 & 1500 & 1500 & 1500 \\
\hline$\Delta t(\mathrm{~s})$ & $\sim 1.5$ & 1.0 & 4.0 & 5.0 \\
\hline$N_{T}$ & $\sim 67000$ & 100000 & 25000 & 20000 \\
\hline$T_{\text {CPU }}^{\prime}(\mathbf{s})$ & 2.0 & 0.94 & 0.25 & 1.32 \\
\hline Computer & DEC 3000 & CRAY-XMP & CRAY-YMP & CRAY-YMP \\
\hline
\end{tabular}

$N_{x}, N_{y}, N_{z}:$ number of points in $x$-, $y$ - and $z$-directions respectively; $L_{x}, L_{y}$ and $L_{z}:$ size of the computational domain; $\Delta t$ : time-step; $N_{T}$ : the total number of time-steps for the total simulation period; $T_{\mathrm{CPU}}$ : CPU time per time-step.

\section{(a) Time series}

Figure 2 shows the vertically integrated TKE (resolved + subgrid). It is clear that the level of TKE is about the same in all runs and that after the first non-dimensional time unit when the resolved scales rapidly amplify, a levelling off of the TKE at

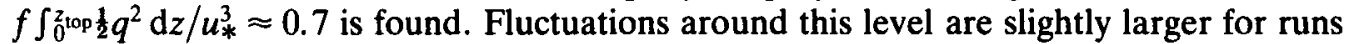
with Moeng's and Nieuwstadt's codes, but the turbulence intensity seems to have reached a quasi-steady state in all codes. The non-backscatter run of Mason-Brown (not shown) has a value of normalized vertically integrated TKE approximately $10 \%$ larger than the backscatter run, and in good agreement with the other runs. $u_{*} / U_{\mathrm{G}}$ is quite constant in all runs, with an average value of 0.0425 for Andrén-Moeng's run, 0.0448 for MasonBrown's backscatter run, 0.0402 for Mason-Brown's non-backscatter run, 0.0402 for Nieuwstadt's run, and 0.0425 for Schumann-Graf's run. The $10 \%$ increase in surface stress found by Mason-Brown when using backscatter is associated with a change in the velocity profile (see below). We also note that a smoother development of the resolved scales during the first non-dimensional time unit takes place when backscatter is included.

Sensitive measures of non-stationarity in the mean fields are given by the quantities

and

$$
C_{u}=-\frac{f}{(\overline{u w})_{0}} \int_{0}^{z_{\text {top }}}\left(\langle\bar{v}\rangle-V_{\mathrm{G}}\right) \mathrm{d} z,
$$

$$
C_{v}=+\frac{f}{(\overline{v w})_{0}} \int_{0}^{z_{\text {top }}}\left(\langle\bar{u}\rangle-U_{\mathrm{G}}\right) \mathrm{d} z,
$$

where $z_{\text {top }}$ is the top of the simulated domain at which the stress-free lid is applied. In an exact steady state situation, both $C_{u}$ and $C_{v}$ should be 1.0. Due to the inertial oscillation, this regime is approached only very slowly, as shown in Figs. 3(a) and (b). We notice that the different runs are closely in phase, as they should be, since they were all initialized in the same way. By averaging over the same time period, we hope to have minimized any differences due to this inertial oscillation, which will show up mainly in the first-order moments. The mean velocity gradients vary only slowly in time and the higher moments which achieve steady state at the turbulence time scale and have been calculated with respect to horizontal averages are less sensitive to this oscillation.

\section{(b) Profiles of fluxes and variances}

Normalized profiles of the first moments, $\langle\bar{u}\rangle,\langle\bar{v}\rangle$, and $\langle\bar{c}\rangle$, appear reasonably similar, 


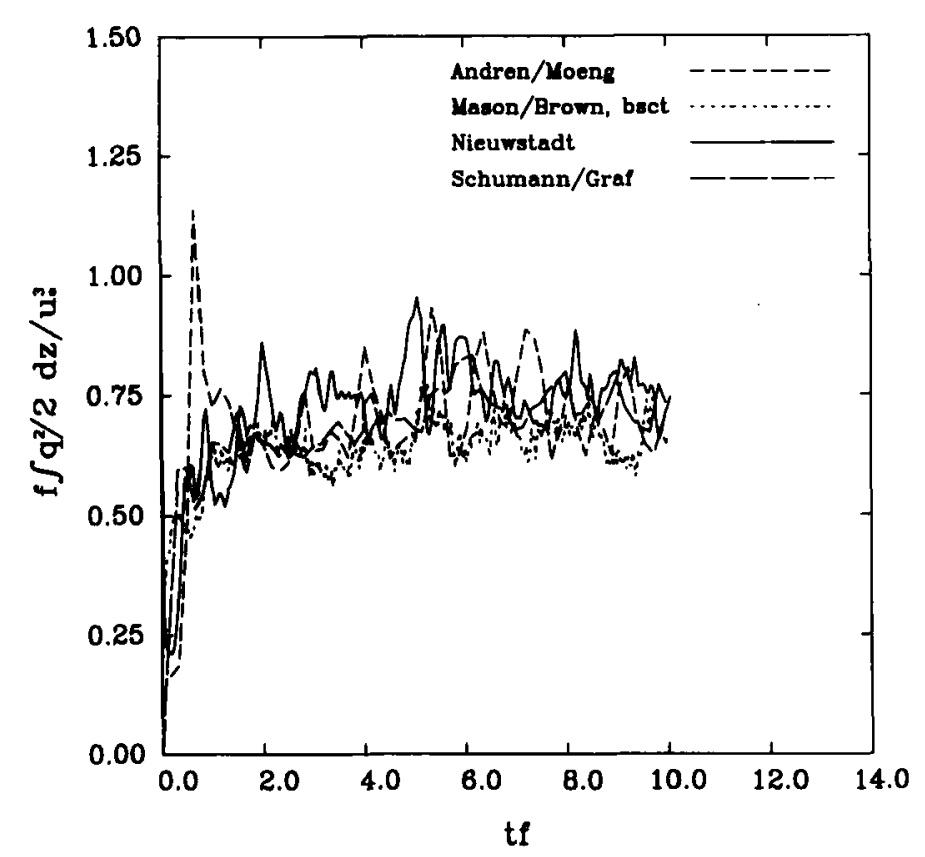

Figure 2. Time series of normalized vertically integrated turbulent kinetic energy. The run by Mason-Brown here is the backscatter case with non-uniform vertical grid. In this and following figures we denote backscatter runs with bsct, and non-backscatter runs with nbsct.

within $\approx 10 \%$ of each other, and are not shown here. However, it is revealing to look at the non-dimensional gradients in the surface layer where, as noted in section 2(a), the traditional Smagorinsky model has consistently failed to produce logarithmic mean profiles. Values of non-dimensional velocity gradient, $\Phi_{M}$, and scalar gradient, $\boldsymbol{\Phi}_{\mathrm{C}}$, defined here as

$$
\Phi_{M}=\frac{K z}{u_{*}} \sqrt{\left(\frac{\partial\langle\bar{u}\rangle}{\partial z}\right)^{2}+\left(\frac{\partial\langle\bar{u}\rangle}{\partial z}\right)^{2}}
$$

and

$$
\Phi_{\mathrm{C}}=\frac{K z}{c_{*}} \frac{\partial\langle\bar{c}\rangle}{\partial z}
$$

should be constant in the logarithmic layer. Profiles of $\Phi_{M}$ and $\Phi_{C}$, Figs. 4(a) and (b), show excessive shear in velocity and scalar fields for all codes used here, except in the backscatter version of Mason's code, which produce a smooth linear transition from the boundary in to the interior of the flow.

Notable differences appear in the velocity variances, which are shown in Fig. 5. We notice that Moeng's and Nieuwstadt's codes give a peak in $\overline{u^{2}} / u_{*}^{2}$ at about $z f / u_{*}=0.03$. Furthermore, at this level, a $50 \%$ difference in total $\overline{u^{2}} / u_{*}^{2}$ is noted among the four results. The non-backscatter runs with Mason's code show similar peaks although they are slightly larger and occur at a lower height. We also notice in both the backscatter and non-backscatter runs with Mason's code that $\overline{u^{2}} / u_{*}^{2}$ is not sensitive to the stretched grid option (comparison not shown). Mason and Thomson (1992) noted a similar reduction in the magnitude of the peak in $u^{2} / u_{*}^{2}$ when using backscatter, and concluded that the sharp peak in $\overline{u^{2}} / u_{*}^{2}$ in their non-backscatter runs was due to the excessive shear in the mean flow in the semi-resolved region. 

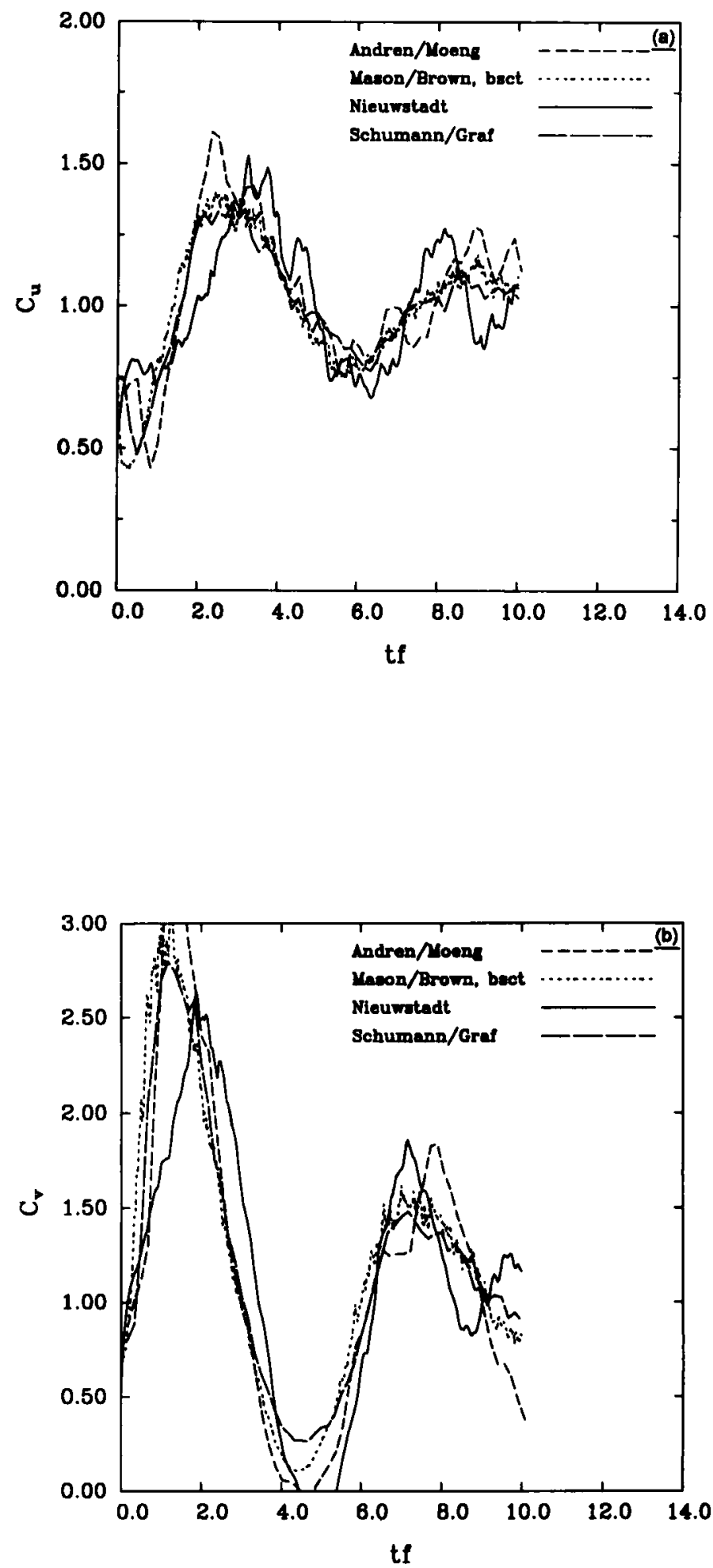

Figure 3. (a) $C_{u}$ of Eq. (6) showing the deviation from steady-state conditions in the $x$-component momentum equation and (b) $C_{v}$ of $\mathrm{Eq}$. (7) showing the deviation from steady-state conditions in the $y$-component momentum equation. 

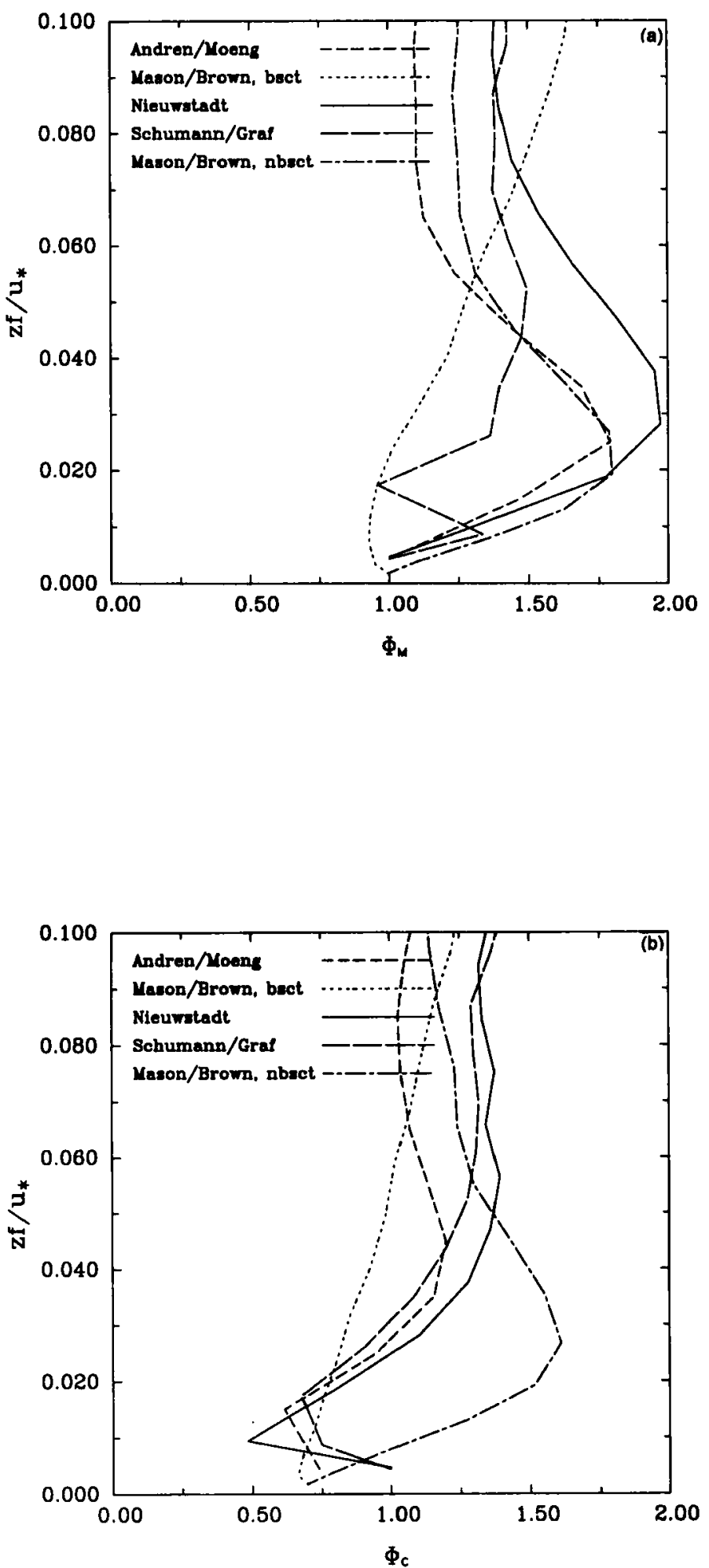

Figure 4. (a) Non-dimensional gradients of mean velocity and (b) non-dimensional gradients of mean scalar fields. 

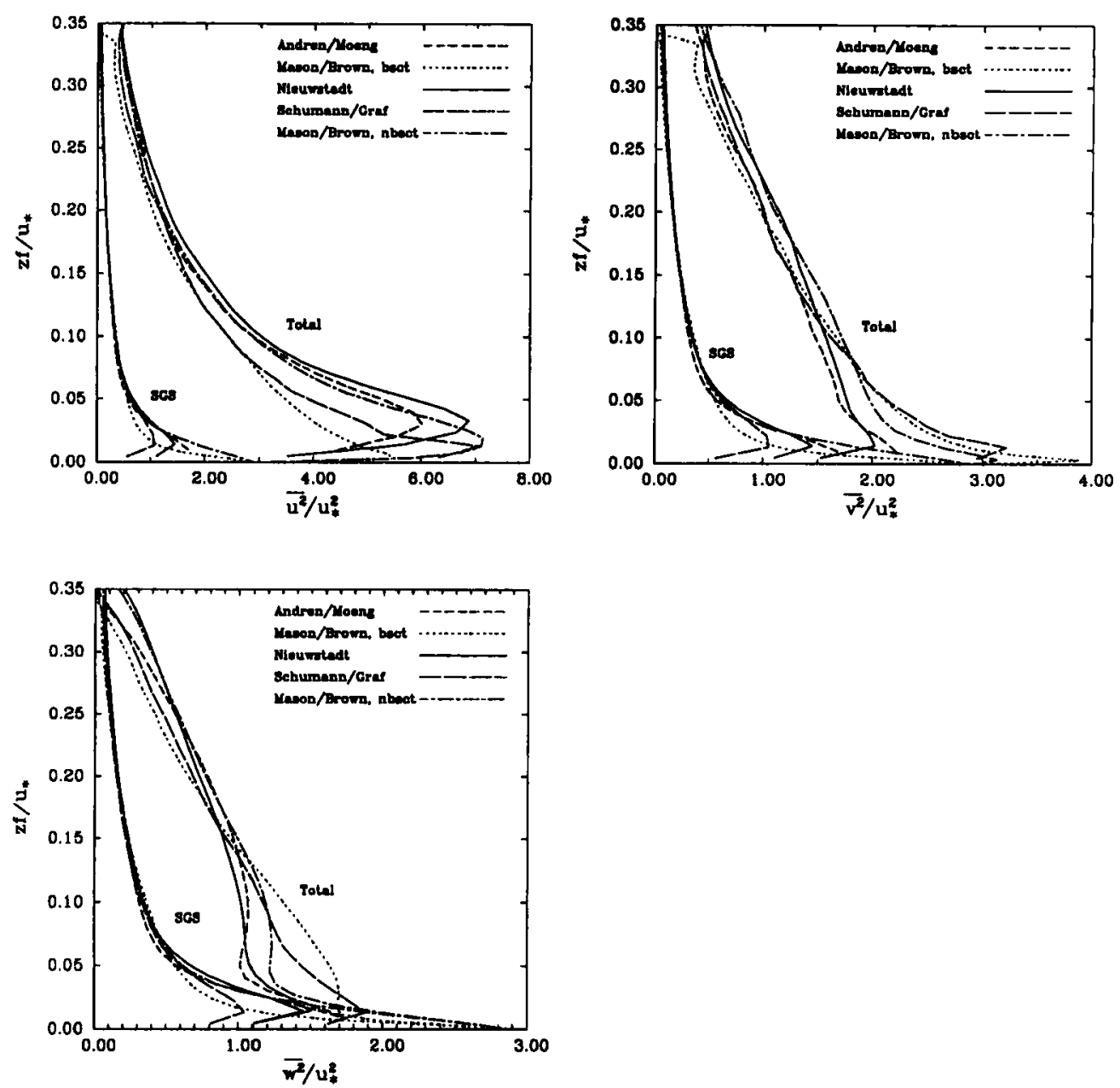

Figure 5. Normalized velocity variances, showing both the sum of resolved and SGS contributions and the SGS part.

On the whole results from Schumann's code are closer to Mason-Brown's backscatter runs. This is particularly noticeable in $\overline{v^{2}} / u_{*}^{2}$, where Schumann's code gives about the same resolved-scale contribution as in the backscatter run with Mason's code. Since the SGS formulation used in Schumann's code for this non-buoyant case is equivalent to what is used in Moeng's and Nieuwstadt's codes, we must attribute the difference to the value of the Smagorinsky constant, $C_{\mathrm{s}}$, and possibly to the differences in the numerical methods. With the coarse resolution used in this simulation, we should expect results to have some sensitivity to SGS parameters, especially near the surface. We notice that the SGS fraction of the TKE agrees well among the different runs, except for the layer below $z f / u_{*}=0.05$. Here we also note a decrease in SGS TKE when backscatter is included in Mason-Brown's code.

Profiles of total vertical fluxes of $u$ - and $v$-momentum agree well among the runs (Figs. 6(a) and (b)), although differences are found in the SGS part near the surface. Results from Moeng's and Nieuwstadt's codes, and from Mason's code without backscatter are similar, whereas Schumann's code gives a very small subgrid scale flux. Mason's code with backscatter lies in between. 

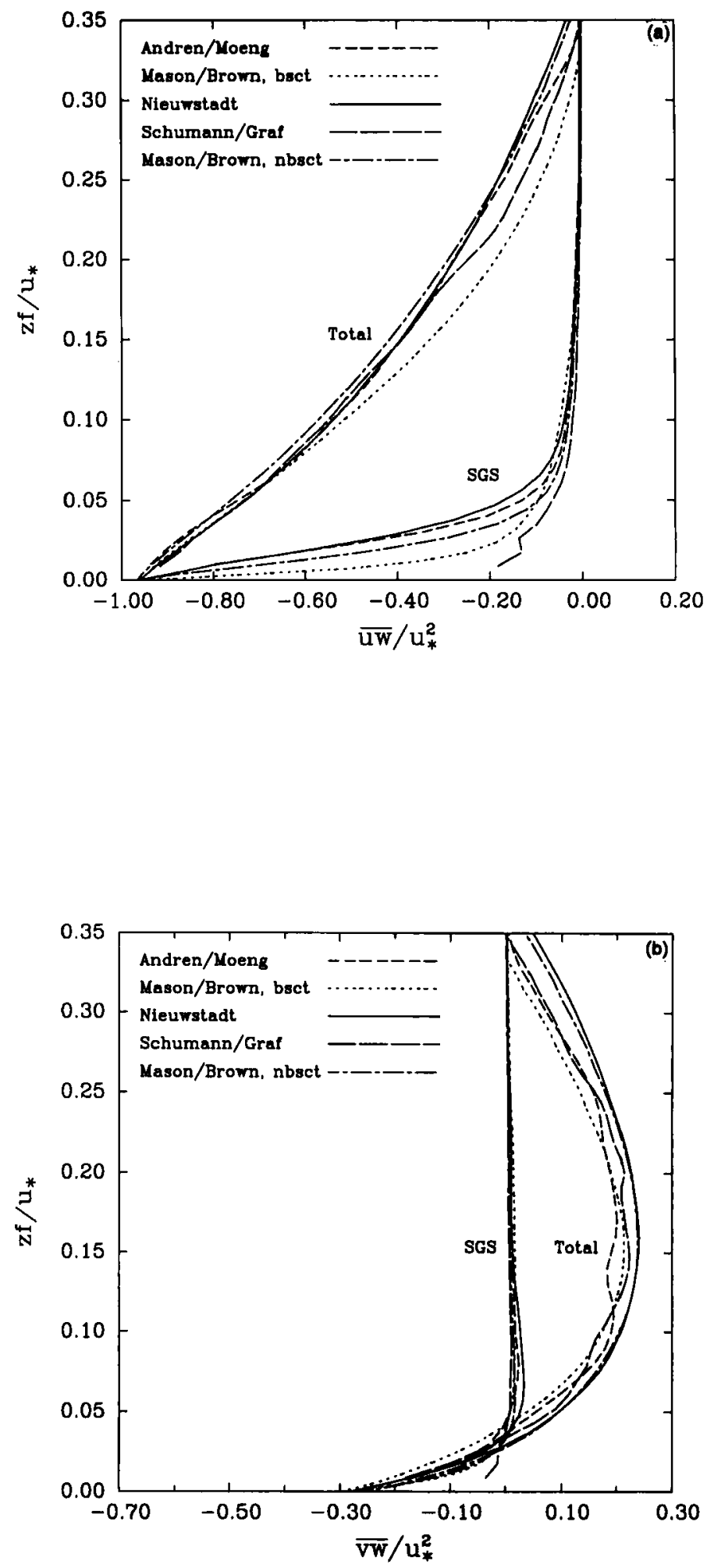

Figure 6. (a) As in Fig. 5 but for the normalized vertical flux of $x$-component momentum and (b) as in Fig. 5 but for the normalized vertical flux of $y$-component momentum. 
Figure 7 shows the subgrid and total variance of the passive scalar, $\overline{c^{2}} / c_{*}^{2}$. The SGS part is roughly the same for all models so that the differences observed in Fig. 7 must be due to the resolved motions. In particular, we notice a faster fall-off of resolved-scale $\overline{c^{2}}$ as the surface is approached in the results of Andrén-Moeng and Nieuwstadt than in those of Mason-Brown and Schumann-Graf. Mason-Brown's non-backscatter level of $\overline{c^{2}} / c_{*}^{2}$ is almost twice as large as the results from Moeng's, Nieuwstadt's and Schumann's codes. This is primarily due to the much larger Schmidt number used by Mason-Brown leading to smaller eddy diffusivities for the scalar. A short test run with reduced Schmidt number confirmed the expected decrease in $\overline{c^{2}} / c_{*}^{2}$. A further test where all codes were run using the same eddy diffusivities will be described in section 5 . The large reduction in $\bar{c}^{2} / c_{*}^{2}$ when backscatter is included is again believed to be linked to the changes in near-surface gradients, in particular to the reduction in mean scalar gradient. The Smolarkiewicz scheme which produces some numerical diffusion may be responsible for slightly smaller scalar variances in Schumann's code.

Figure 8 shows $\overline{w c} / u_{*} c_{*}$ and we note significant differences in the magnitude of the SGS part between different codes, as we did for the vertical momentum flux, reflecting the differences in SGS eddy diffusivities and Schmidt numbers. Again Schumann's code gives a SGS contribution of about the same magnitude as that obtained from the backscatter runs with Mason's code. (Note that differences in $u_{*}$ between the runs puts the top of the domain at different $z f / u_{*}$ in this normalized representation.)

We now direct our attention to the pressure-related correlations. $\bar{p}_{\mathrm{r}}$ in Eq. (1) represents only the resolved-scale pressure. In later figures, however, we will consider one third of the trace of the SGS stress tensor, i.e. $\frac{2}{3} e$, into the $\bar{p}$ notation, so that $\bar{p}=\bar{p}_{\mathrm{r}}+\frac{2}{3} \rho e$. By doing so, we avoid subtraction of the estimated subgrid energy in Mason's code where the SGS energy is not explicitly calculated. Also, the backscatter formulation in Mason's code does not explicitly cover the irrotational part of the random

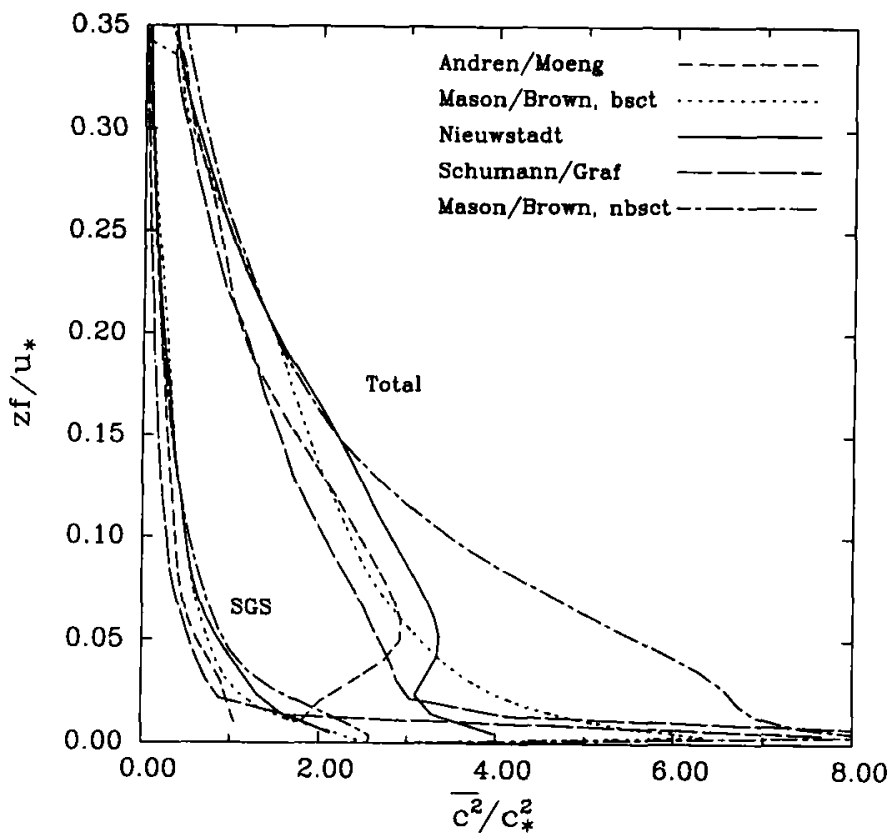

Figure 7. As in Fig. 5 but for normalized scalar variance. 


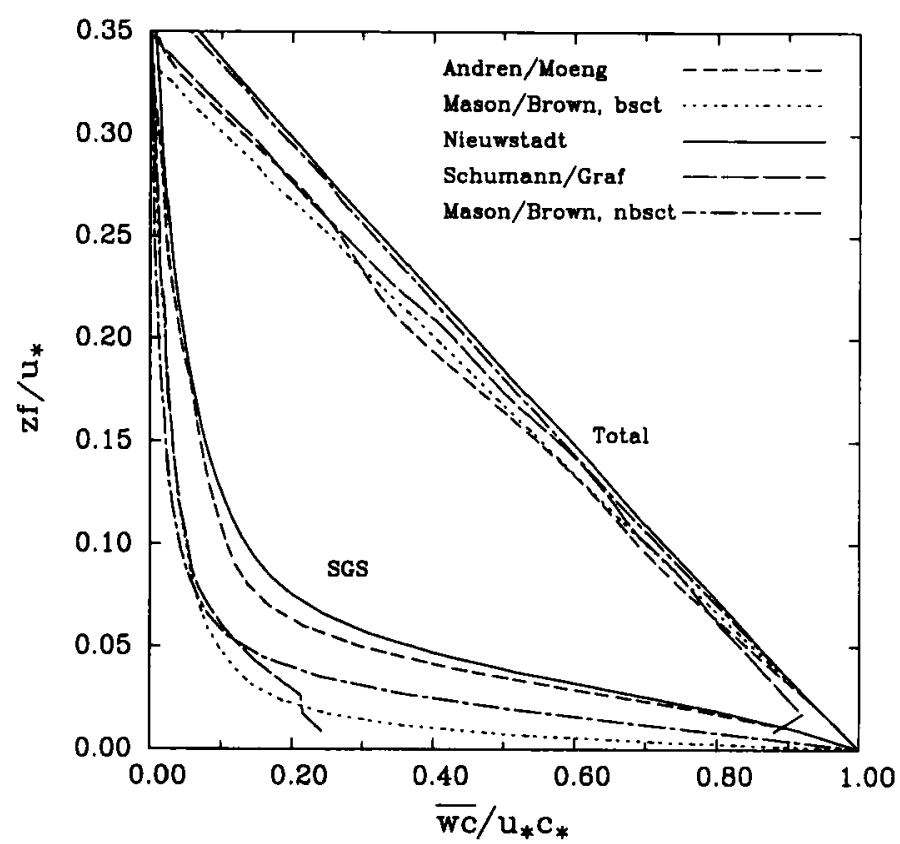

Figure 8. As in Fig. 5 but for the $z$-component scalar flux.

stresses preventing extraction of true pressure from the backscatter runs. Since pressure, through its dependency on integrals of velocity products, is a second-order quantity, we expect larger scatter due to sampling. This is corroborated by, e.g., $\overline{w p} / \rho u_{*}^{3}$, in Fig. 9, where the shaded region shows the envelope of plus and minus one standard deviation from the 18 instantaneous profiles of Andren-Moeng's data. The width of the shaded area in Fig. 9 indicates that in order to get reliable results for the pressure correlations, one needs significantly better resolution and a larger data set size than used here. Our use of a rather coarse mesh means that a significant part of the correlation comes from the ${ }_{3}^{2} \rho e$ term. For example, if we use only resolved-scale pressure, $p_{\mathrm{r}}$, in the data from Moeng's code, we obtain roughly twice as large a peak in the lower boundary layer, in accord with previous high-resolution Ekman layer simulations with Moeng's code (Andrén and Moeng 1993).

For the standard deviation of pressure, $\left(\overline{p^{2}}\right)^{1 / 2} / \rho u_{*}^{2}$, Fig. 10, we find quite varying vertical structures in lower levels. Schumann-Graf's run, which has the smallest SGS eddy diffusivity, and Mason-Brown's backscatter run both show a continuous increase of $\left(p^{2}\right)^{1 / 2} / \rho u_{*}^{2}$, all the way down to the surface. As will be shown later in section 5 these differences largely disappear if the LES codes are run with the same SGS eddy diffusivity profile. We notice that the difference between backscatter and non-backscatter runs is about $20 \%$ in the interior of the flow. An analysis of the results from the simulation with Moeng's code shows that the inclusion of $\frac{2}{3} \rho e$ in the pressure variable changes $\left(\overline{p^{2}}\right)^{1 / 2} / \rho u_{*}^{2}$, by only about $5 \%$. We tentatively conclude that inclusion of backscatter has a non-negligible impact on the pressure field even in the interior of the flow of a coarse resolution LES. Our results thus confirm the expected sensitivity of coarse LES results to the Smagorinsky constant as well as to the backscatter effect. 


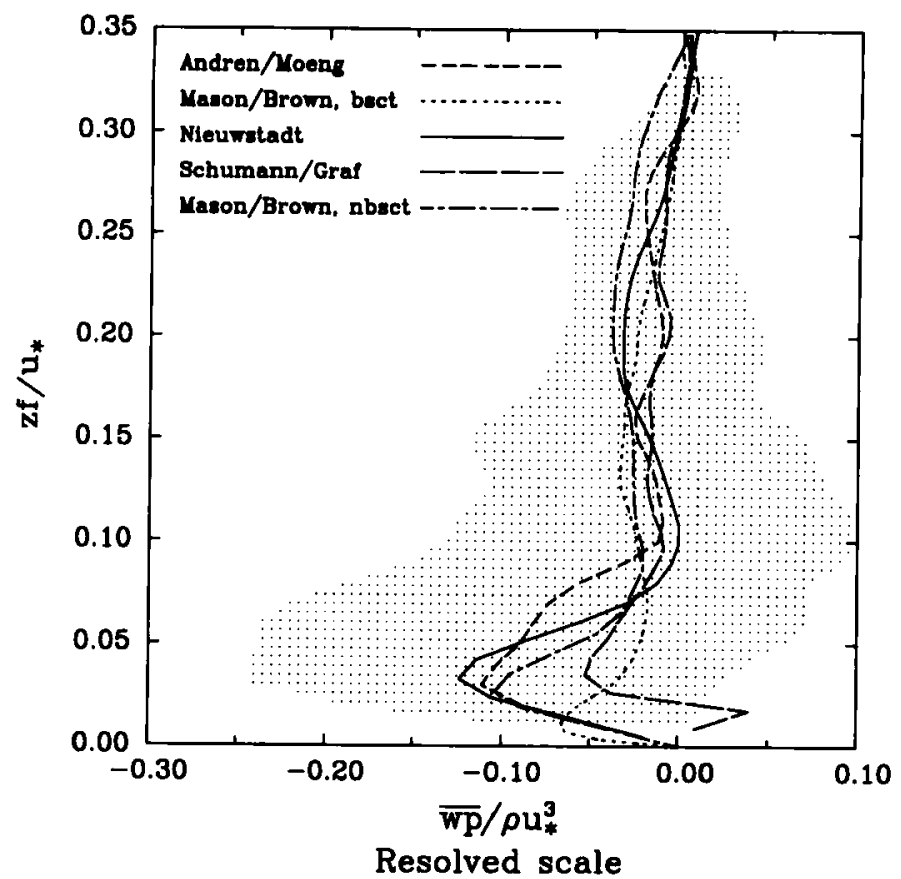

Figure 9. Normalized resolved-scale $z$-component pressure flux, where $\bar{p}=\bar{p}_{\mathrm{r}}+\xi_{3} \rho e$ as defined in the text.

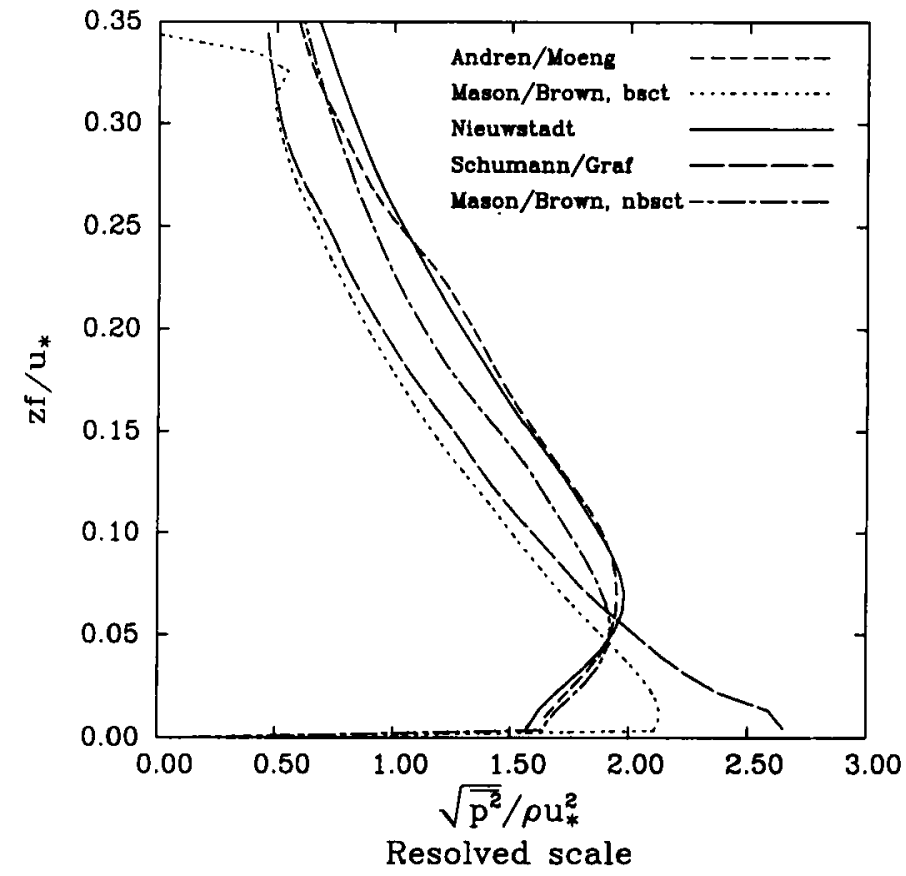

Figure 10. Normalized standard deviation of pressure $\bar{p}$, where $\bar{p}=\bar{p}_{\mathrm{r}}+3 \rho$ as defined in the text. 


\section{(c) Budgets of TKE and vertical fluxes}

We limit our analysis of budgets to the resolved-scale TKE and vertical fluxes of momentum and scalars. We stress that our budgets are for the resolved-scale parts only and that the pressure effect is included in the form of $\bar{p}=\bar{p}_{\mathrm{r}}+\frac{2}{3} \rho e$. The inclusion of $\frac{2}{3} e$ in the definition of pressure completely alters the vertical distributions of the pressure terms in the flux budgets as compared to using just $\bar{p}_{\mathrm{r}}$ in these coarse-resolution simulations. Our budget equations are for the vertical flux of $u$-momentum

$$
\begin{gathered}
\frac{\partial}{\partial t}\left\langle\bar{u}^{\prime \prime} \bar{w}^{\prime \prime}\right\rangle=-\left\langle\bar{w}^{\prime \prime} \bar{w}^{\prime \prime}\right\rangle \frac{\partial\langle\bar{u}\rangle}{\partial z}-\frac{\partial}{\partial z}\left\langle\bar{u}^{\prime \prime} \bar{w}^{\prime \prime} \bar{w}^{\prime \prime}\right\rangle \\
\text { Tendency } \\
\begin{array}{c}
\text { Production } \\
\text { Turbulent transport }
\end{array} \\
-\frac{1}{\rho_{0}}\left\langle\bar{w}^{\prime \prime} \frac{\partial}{\partial x}\left(\bar{p}_{\mathrm{r}}+\frac{2}{3} \rho e\right)+\bar{u}^{\prime \prime} \frac{\partial}{\partial z}\left(\bar{p}_{\mathrm{r}}+\frac{3}{3} \rho e\right)\right\rangle \\
+\left\langle\bar{w}^{\prime \prime}\left(\frac{\partial}{\partial x}\left(2 K_{\mathrm{M}} \frac{\partial \bar{u}}{\partial x}\right)+\frac{\partial}{\partial y}\left(K_{\mathrm{M}}\left(\frac{\partial \bar{u}}{\partial y}+\frac{\partial \bar{v}}{\partial x}\right)\right)+\frac{\partial}{\partial z}\left(K_{\mathrm{M}}\left(\frac{\partial \bar{u}}{\partial z}+\frac{\partial \bar{w}}{\partial x}\right)\right)\right)+\right. \\
\left.\bar{u}^{\prime \prime}\left(\frac{\partial}{\partial x}\left(K_{\mathrm{M}}\left(\frac{\partial \bar{w}}{\partial x}+\frac{\partial \bar{u}}{\partial z}\right)\right)+\frac{\partial}{\partial y}\left(K_{\mathrm{M}}\left(\frac{\partial \bar{w}}{\partial y}+\frac{\partial \bar{v}}{\partial z}\right)\right)+\frac{\partial}{\partial z}\left(2 K_{\mathrm{M}} \frac{\partial \bar{w}}{\partial z}\right)\right)\right\rangle \\
\text { Subgrid scale } \\
-\hat{f}\left\langle\bar{w}^{\prime \prime} \bar{w}^{\prime \prime}\right\rangle+\hat{f}\left\langle\bar{u}^{\prime \prime} \bar{u}^{\prime \prime}\right\rangle+f\left\langle\bar{v}^{\prime \prime} \bar{w}^{\prime \prime}\right\rangle, \\
\text { Coriolis }
\end{gathered}
$$

and for the vertical flux of a passive scalar

$$
\begin{gathered}
\frac{\partial}{\partial t}\left\langle\bar{w}^{\prime \prime} \bar{c}^{\prime \prime}\right\rangle=-\left\langle\bar{w}^{\prime \prime} \bar{w}^{\prime \prime}\right\rangle \frac{\partial\langle\bar{c}\rangle}{\partial z}-\frac{\partial}{\partial z}\left\langle\bar{w}^{\prime \prime} \bar{c}^{\prime \prime} \bar{w}^{\prime \prime}\right\rangle \\
\text { Tendency } \quad \text { Production } \quad \text { Turbulent transport } \\
-\frac{1}{\rho_{0}}\left\langle\bar{c}^{\prime \prime} \frac{\partial}{\partial z}\left(\bar{p}_{\mathrm{r}}+\frac{2}{3} \rho e\right)\right\rangle \\
+\left\langle\bar{w}^{\prime \prime}\left(\frac{\partial}{\partial x}\left(K_{\mathrm{C}} \frac{\partial \bar{c}}{\partial x}\right)+\frac{\partial}{\partial y}\left(K_{\mathrm{C}} \frac{\partial \bar{c}}{\partial y}\right)+\frac{\partial}{\partial z}\left(K_{\mathrm{C}} \frac{\partial \bar{c}}{\partial z}\right)\right)+\right. \\
\left.\bar{c}^{\prime \prime}\left(\frac{\partial}{\partial x}\left(K_{\mathrm{M}}\left(\frac{\partial \bar{w}}{\partial x}+\frac{\partial \bar{u}}{\partial z}\right)\right)+\frac{\partial}{\partial y}\left(K_{\mathrm{M}}\left(\frac{\partial \bar{w}}{\partial y}+\frac{\partial \bar{v}}{\partial z}\right)\right)+\frac{\partial}{\partial z}\left(2 K_{\mathrm{M}} \frac{\partial \bar{w}}{\partial z}\right)\right)\right\rangle \\
\text { Subgrid scale } \\
+\hat{f}\left(\bar{u}^{\prime \prime} \bar{c}^{\prime \prime}\right\rangle, \\
\text { Coriolis }
\end{gathered}
$$

where $\bar{u}^{\prime \prime}=\bar{u}-\langle\bar{u}\rangle$ and $\hat{f}$ is the horizontal Coriolis component.

In the TKE budgets we find the expected balance between production and SGS effects in all simulations, all other terms are negligibly small. Therefore we will show only the dissipation rate profile of the TKE budget. Profiles of the dissipation of resolvedscale TKE, Fig. 11, show a good agreement in the interior of the flow. Differences are found below $z f / u_{*}=0.05$, in the surface layer. Codes using smaller effective SGS eddy diffusivities generally show larger values of the dissipation term in this layer. The increase in resolved-scale strain rates thus dominates over the decrease in SGS eddy diffusivities 


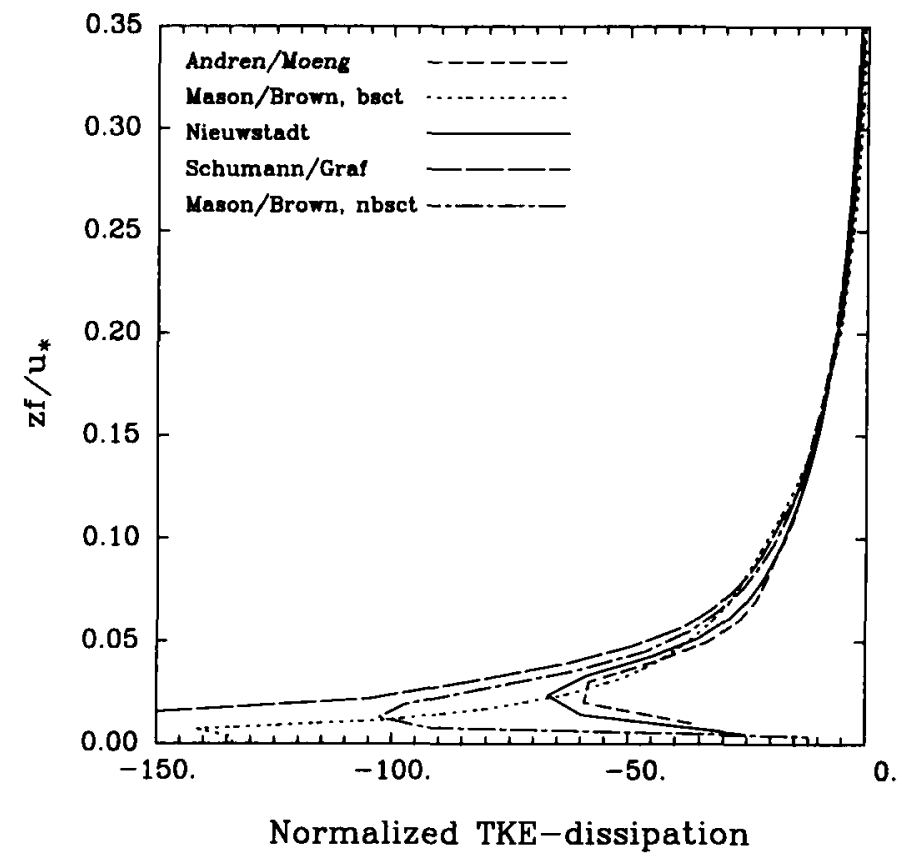

Figure 11. Normalized profiles of dissipation of resolved-scale TKE.

such that the net dissipation of the normalized resolved-scale TKE is increased toward the surface.

Budgets of the resolved-scale vertical flux of $u$-momentum in Fig. 12 also show negligible tendency terms. The flux budgets are dominated by production and pressure terms. Coriolis and turbulent transport terms are minor, except for Schumann-Graf's results where the transport term makes a non-negligible contribution. Moeng's and Nieuwstadt's codes and Mason's code without backscatter give a small SGS term, leaving a close balance between the pressure and the production terms. However, in MasonBrown's backscatter run, the SGS term becomes a non-negligible contribution, so too in Schumann-Graf's run. This results from the increased turbulence intensity at small scales in these runs. Comparing the non-backscatter with the backscatter case we find that the increase in the magnitude of the production that follows from the increase in resolvedscale $\overline{w w}$ in the backscatter run is balanced by an increase in the SGS term. The pressure term agrees well between the runs in the interior of the flow but is much larger next to the surface in Mason-Brown's backscatter run and Schumann-Graf's run, as compared to other runs. Again this is consistent with these runs having more intense resolved-scale motions close to the surface.

Finally, we stress again that using only resolved-scale pressure, $\bar{p}_{\mathrm{r}}$, in the pressure term, as opposed to the use of $\bar{p}_{\mathrm{r}}+\frac{2}{3} \rho e$. we obtain a radically different picture with a large increase in the SGS effect and also with a corresponding change in the pressure term as the surface is approached.

Results for the budgets of the resolved-scale vertical flux of $v$-momentum are generally similar to the results for the vertical flux of $u$-momentum and are not shown.

Budget differences similar to those for the $\left\langle\bar{u}^{\prime \prime} w^{\prime \prime}\right\rangle$ budgets are found in the budgets of the vertical flux of scalar, $\left\langle\bar{w}^{\prime \prime} c^{\prime \prime}\right\rangle$, Fig. 13. Again, the budgets from Moeng's and 

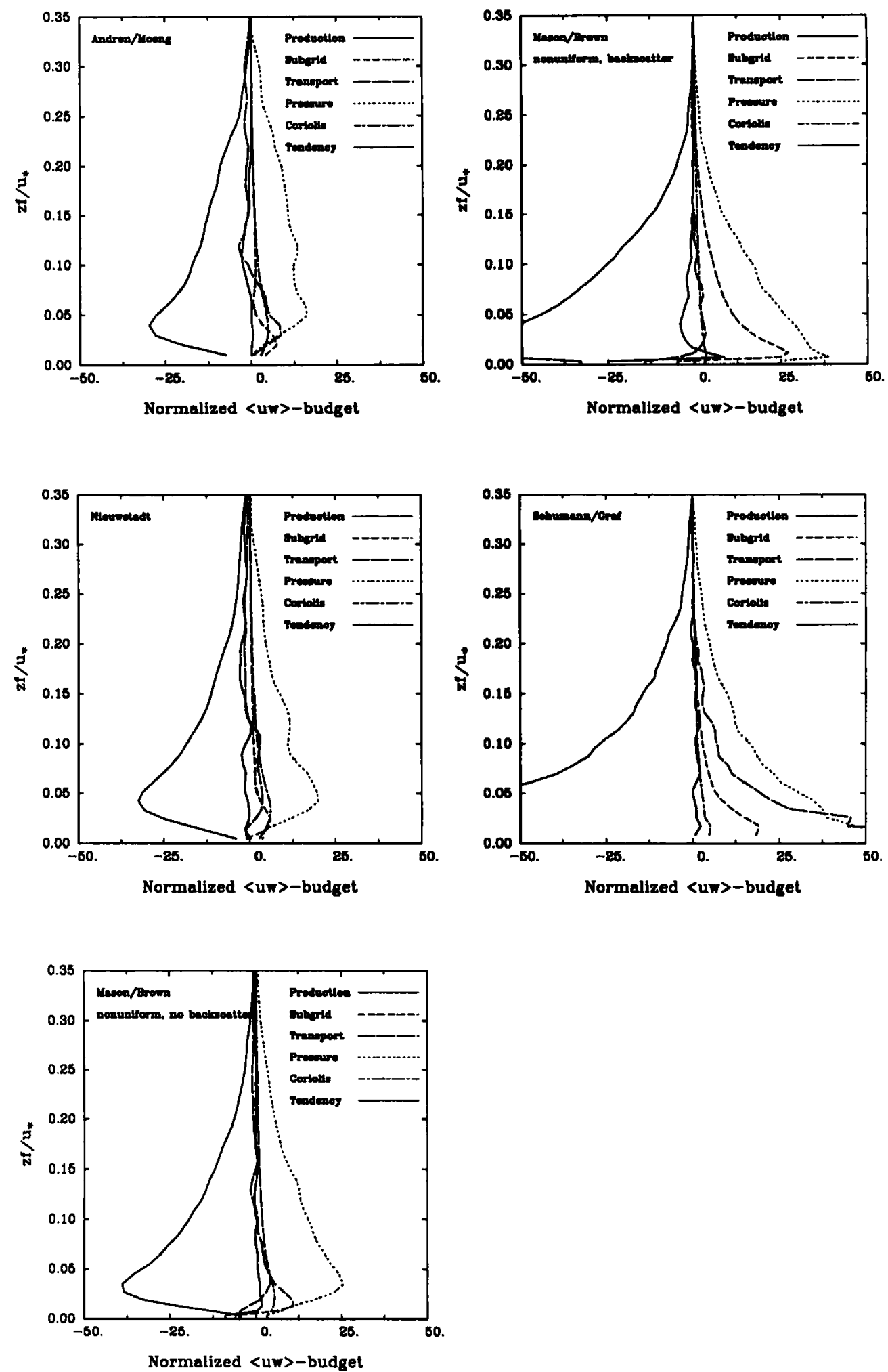

Figure 12. Normalized budgets for resolved-scale vertical flux of $x$-component momentum. 

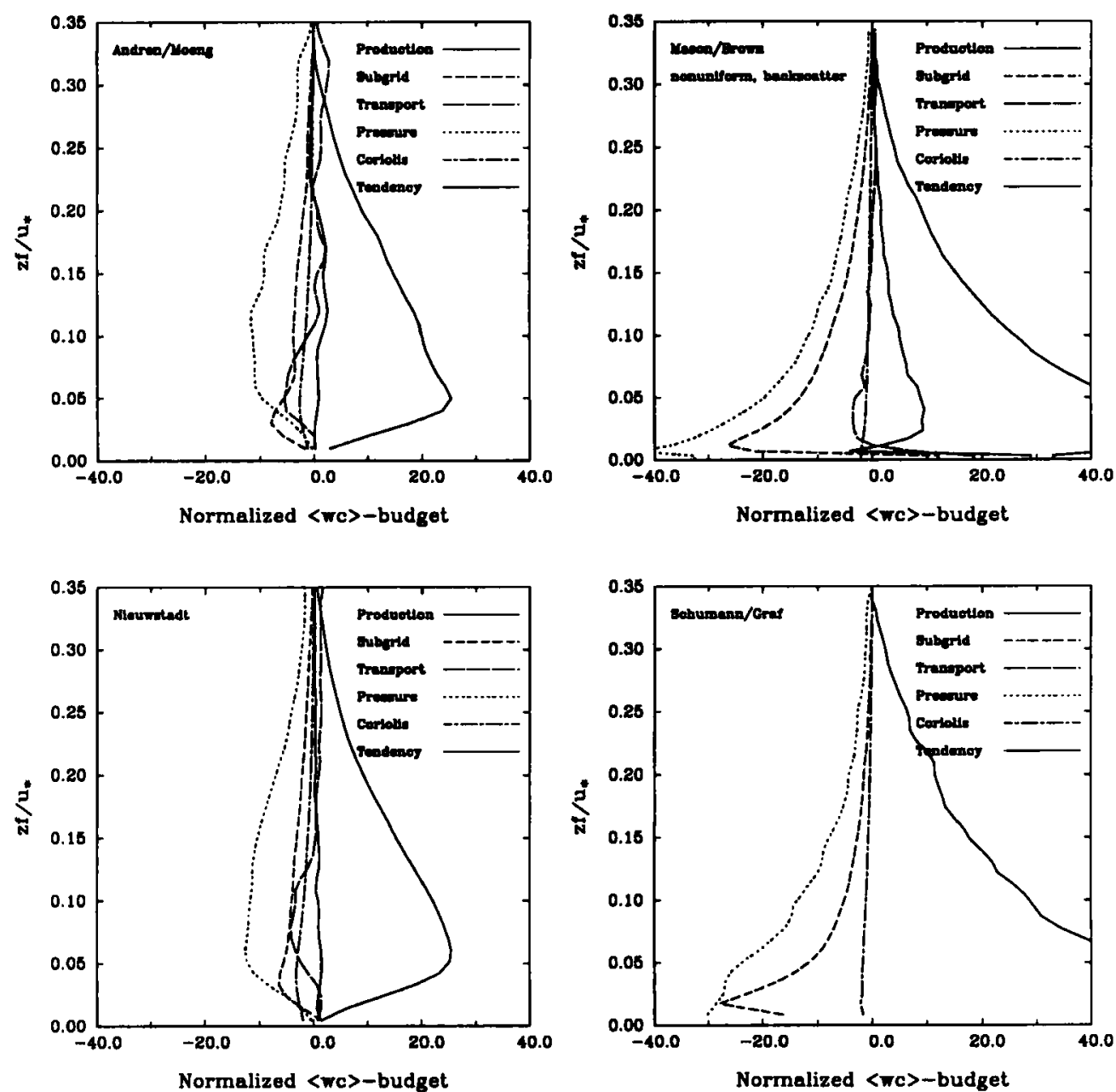

Figure 13. Normalized budget for resolved-scale vertical flux of the passive scalar. The curves for transport and tendency are not shown for Schumann's code because it turned out to be difficult to compute these terms correctly in this code which uses the Smolarkiewicz scheme.

Nieuwstadt's codes agree with each other, showing basically a balance between production and pressure terms with some minor influence of the subgrid terms. (Note again that a completely different picture emerges if one uses only resolved-scale pressure, $p_{\mathrm{r}}$, for the pressure variable. SGS effects are then, together with production and pressure redistribution, the leading terms.) Mason's code without backscatter (not shown) gives a vertical structure similar to Moeng's and Nieuwstadt's codes, but with about $50 \%$ larger magnitudes for production and pressure terms. Schumann-Graf's results are close to Mason-Brown's backscatter results. Note that the transport and tendency terms are not shown for Schumann's code because it turned out to be difficult to compute these terms correctly in this code which uses the Smolarkiewicz advection scheme for scalars. Due to the smaller SGS eddy viscosities, $K_{\mathrm{M}}$ (Fig. 14(a)), and larger Schmidt numbers, the SGS diffusivities for scalars, $K_{\mathrm{C}}$, in Schumann-Graf's and Mason-Brown's runs are only about $50 \%$ of the $K_{\mathrm{C}}$ in Andrén-Moeng's and Nieuwstadt's runs, as shown in Fig. 14(b). Accordingly, we find quite large differences in the budgets. In the Schumann-Graf run 

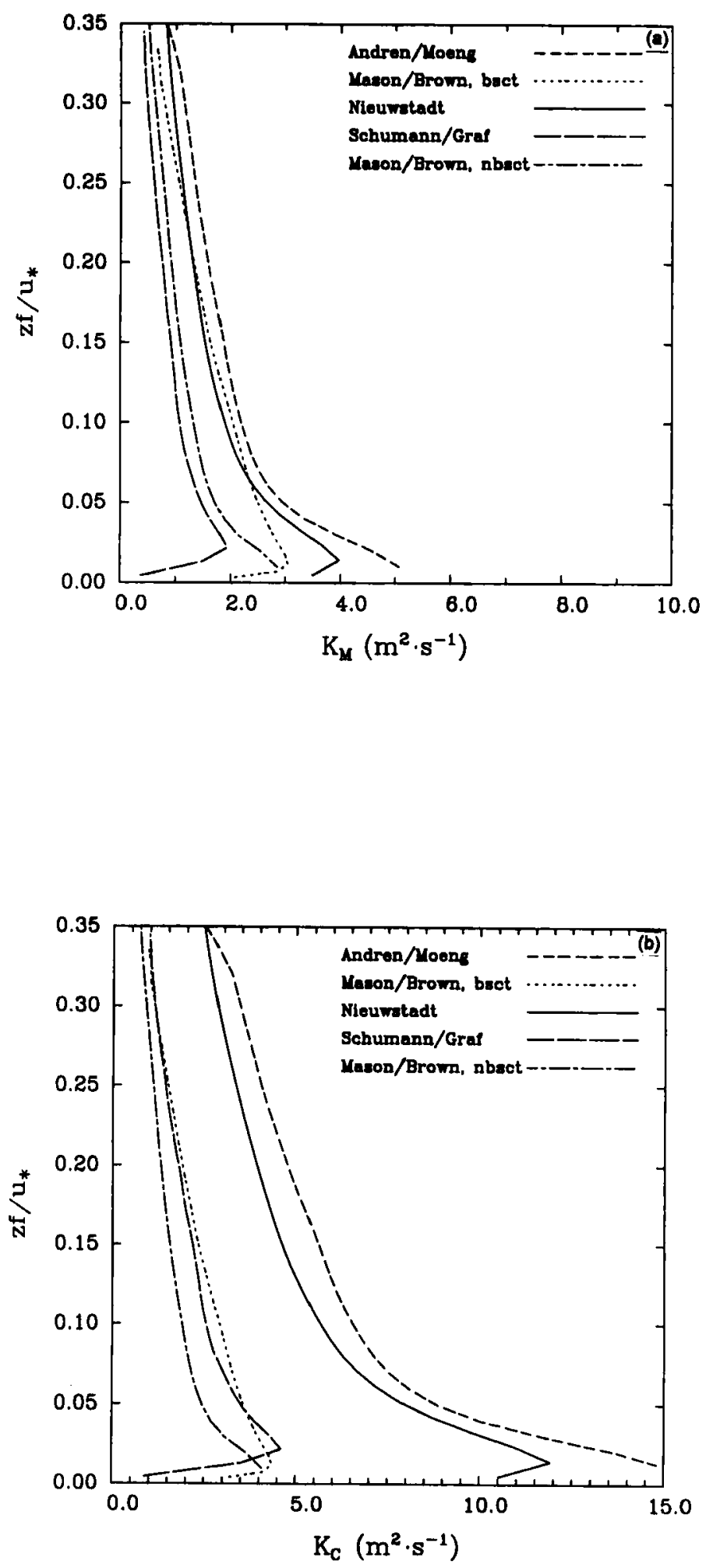

Figure 14. (a) Horizontally averaged SGS eddy diffusivity for momentum, $K_{\mathrm{M}}$ and (b) horizontally averaged SGS eddy diffusivity for scalar, $\boldsymbol{K}_{\mathrm{C}}$. 
and the Mason-Brown backscatter run we find that the production, pressure, and SGS terms all dominate and continue to increase all the way down to the surface. This is in line with differences shown earlier for profiles of correlations involving the scalar fields.

\section{(d) Spectra}

We have calculated energy spectra of the velocity and scalar fields and show here spectra at $z f / u_{*}=0.10$ in Fig. 15. These spectra are calculated from one-dimensional Fourier transformations that are then averaged in the horizontal direction perpendicular to the wavenumber vector component, and in time. We limit the presentation to spectra along the $x$ direction. Note that spectral estimates have been multiplied by a factor of 1000 in order to provide more reasonable labels along the ordinate. These spectra show that smaller SGS eddy diffusivities push spectral peaks towards higher wavenumbers. Results from Moeng's and Nieuwstadt's codes have spectral peaks at nondimensional

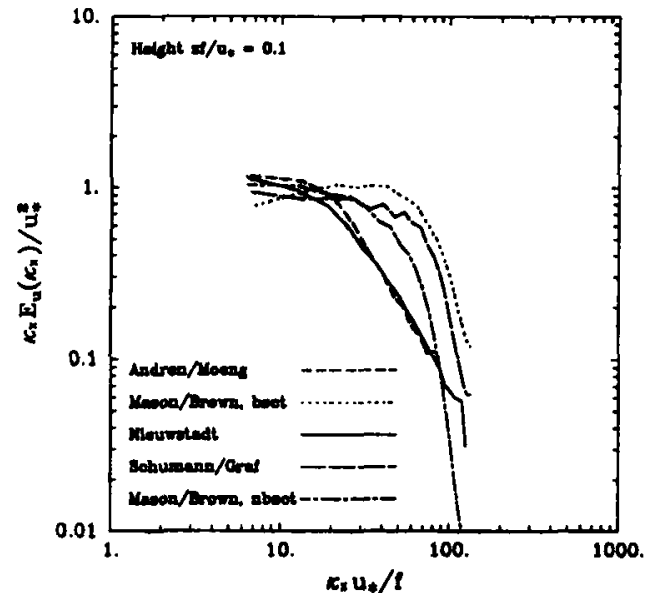

Horizontal enerey pectrum of u

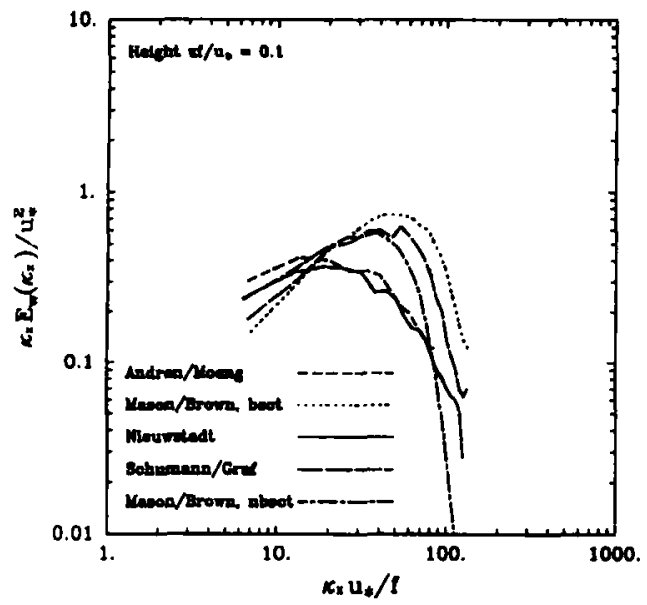

Horizontal energy epectrum of $w$

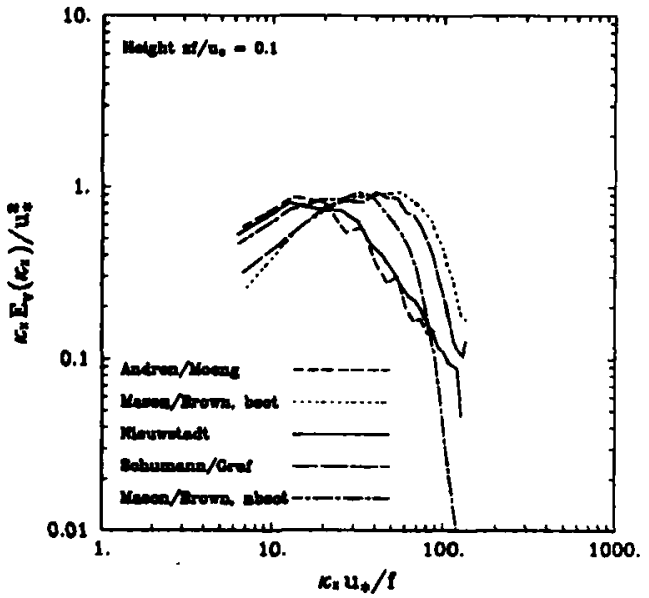

Horizontal energy spectrum of $v$

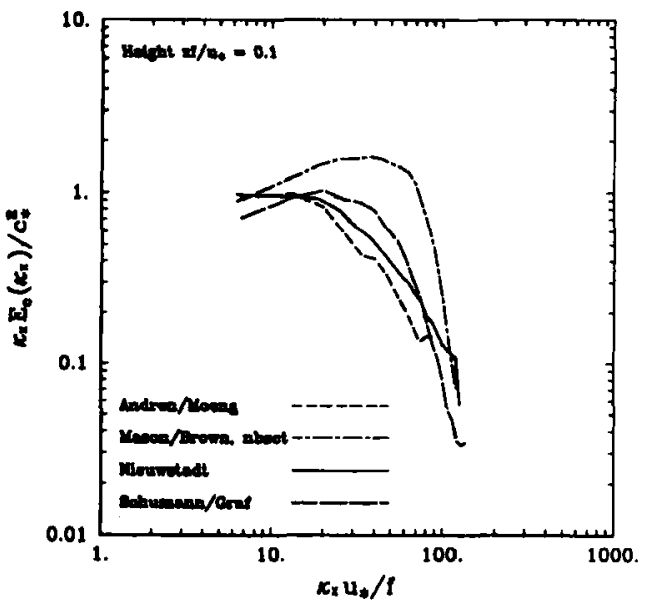

Horizontal energy spectrum of $c$

Figure 15. Normalized spectra at a height of $z f / u_{*}=0.1$ of resolved-scale velocities and passive scalar. Spectral estimates have been multiplied by a factor of 1000 in order to provide more reasonable labels along the ordinate. 
wavenumbers around 20 whereas spectra from Mason's and Schumann's codes peak at non-dimensional wavenumbers around 30-40.

Spectra of the passive scalar indicate the dispersive property of the Smolarkiewicz advection scheme used in Schumann's code. Although the SGS diffusivity for scalars, $K_{\mathrm{C}}$, in Schumann-Graf's run is much closer to Mason-Brown's than to Andrén-Moeng's and Nieuwstadt's, as shown in Fig. 14(b), still Schumann-Graf's scalar spectra are closer to Andrén-Moeng's and Nieuwstadt's than to Mason-Brown's.

Values of SGS eddy diffusivities in Figs. 14(a) and (b) tend to form two groups with Mason-Brown's and Schumann-Graf's showing smaller values than Andrén-Moeng's and Nieuwstadt's. When comparing spectra we notice that spectra from the former two runs tend to have steeper fall-off at higher wavenumbers. As one decreases SGS eddy diffusivities spectra are expected to become increasingly flat, with a drop at the cut-off wavenumber. Thus it seems as if a gradual decrease in SGS eddy diffusivities first moves the spectral peak to higher wavenumbers, with a steeper spectral roll-off at the highest wavenumbers as a result, and then flattens the spectrum. Due to the coarse grid resolution used, none of the runs shows a well defined inertial subrange. This is particularly the case at low levels where the decrease in discrete spectral intensity at higher wavenumbers is significantly faster than $-2 / 3$.

Comparing backscatter with non-backscatter runs from Mason's code, we find the expected shift of spectral peaks to higher wavenumbers when backscatter is included. This results from the continuous random excitation of the smallest resolved scales. A similar effect is obtained with reduced values of SGS eddy diffusivities. By reducing SGS $K_{\mathrm{M}}$ and $K_{\mathrm{C}}$, one effectively reduces the energy dissipation out of the high wavenumbers of resolved fields. With the same energy cascade from larger eddies, this results in more energy in the higher wavenumbers of the resolved fields.

\section{Discussion}

The differences among the four LES codes can only be due to the differences in their SGS eddy diffusivities and their numerics. It follows from Eqs. (1)-(3) that if the SGS eddy diffusivities are fixed only numerical differences will influence the resolvedscale quantities. To isolate these numerical effects all codes were run with fixed SGS diffusivities, $K_{\mathrm{M}}$ and $K_{\mathrm{C}}$, which are set equal to given profiles (and allowed for no backscatter in Mason's model). For this purpose, we have chosen the average $K$-profiles from Mason-Brown's non-backscatter run with uniform grid, shown in Figs. 14(a) and (b). A short test period was used instead of rerunning the whole simulation. Starting from the individual results at $t=7 / f$ and integrating to $t=9 / f$, the last non-dimensional time unit was used for averaging. The fixed profiles of SGS $K_{\mathrm{M}}$ and $K_{\mathrm{C}}$ were also used in the SGS TKE equation used by Andrén-Moeng, Nieuwstadt, and Schumann-Graf.

Figures 16(a) and (b) show that the variances $u^{2} / u_{*}^{2}$ and $\overline{c^{2}} / c_{*}^{2}$ agree very well among the different codes. The spread in profile shapes of $\left(\overline{p^{2}}\right)^{1 / 2} / \rho u_{*}^{2}$ has to a large extent disappeared, as indicated in Fig. 17, such that they closely resemble each other. However, a $20 \%$ lower $\left(\overline{p^{2}}\right)^{1 / 2} / \rho u_{*}^{2}$ level is obtained in Schumann-Graf's test run. The reason for this is not clear. The diffusive properties of the Smolarkiewicz advection scheme affects SGS TKE, but this is likely of secondary importance for $\left(\overline{p^{2}}\right)^{1 / 2} / \rho u_{*}^{2}$. The impact of the SGS model on our previous results is also revealed by the change in the TKE dissipationrate profiles, as shown in Fig. 18, for which we now find the four profiles from all codes more or less on top of each other.

The spectra are particularly interesting since they directly gave us the response of different scales to differences in the numerics. As described in section 2, the numerical 

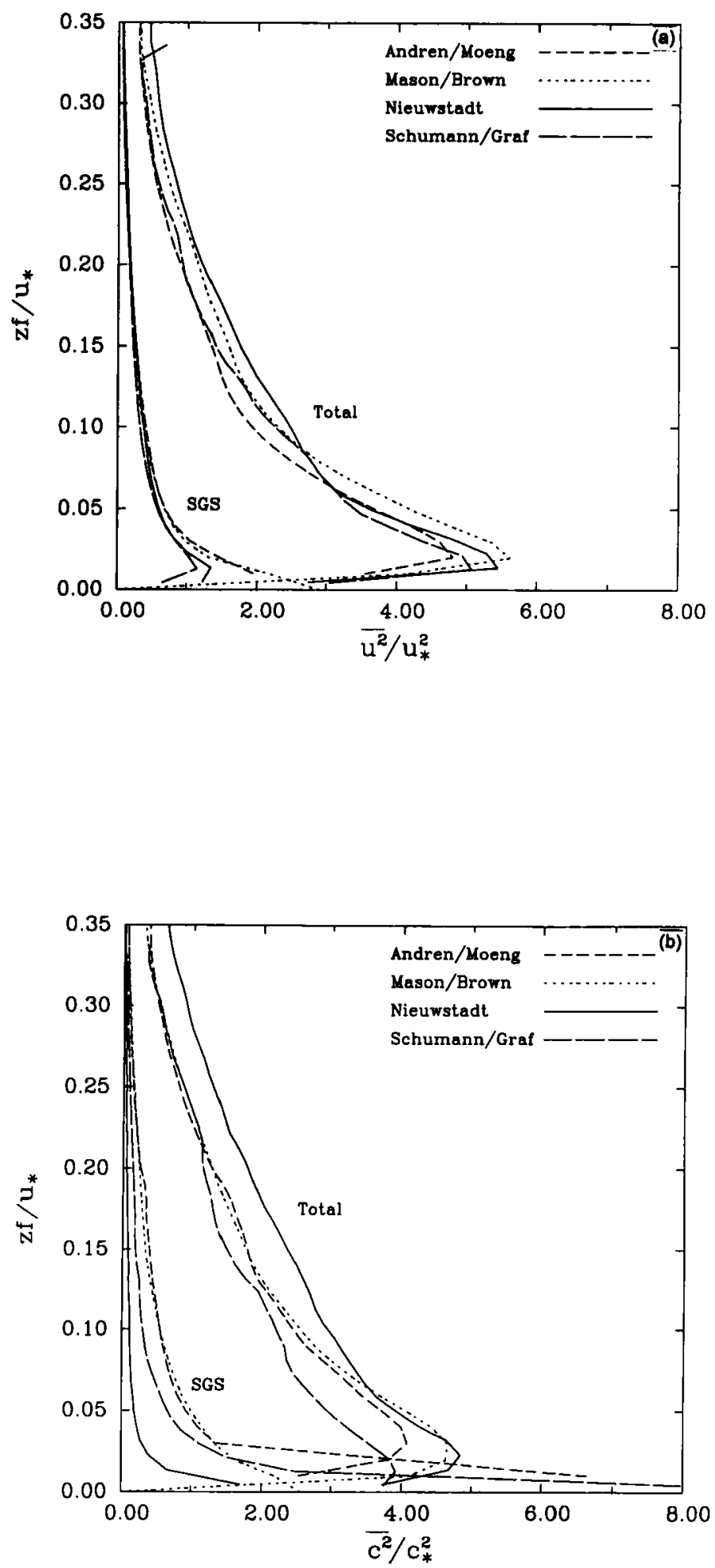

Figure 16. (a) Normalized $x$-component velocity variances from the test run with fixed profiles of SGS eddy diffusivities. The run by Mason-Brown is on a uniform vertical grid, without backscatter. (b) As in Fig. 16(a) but for the scalar variance. 


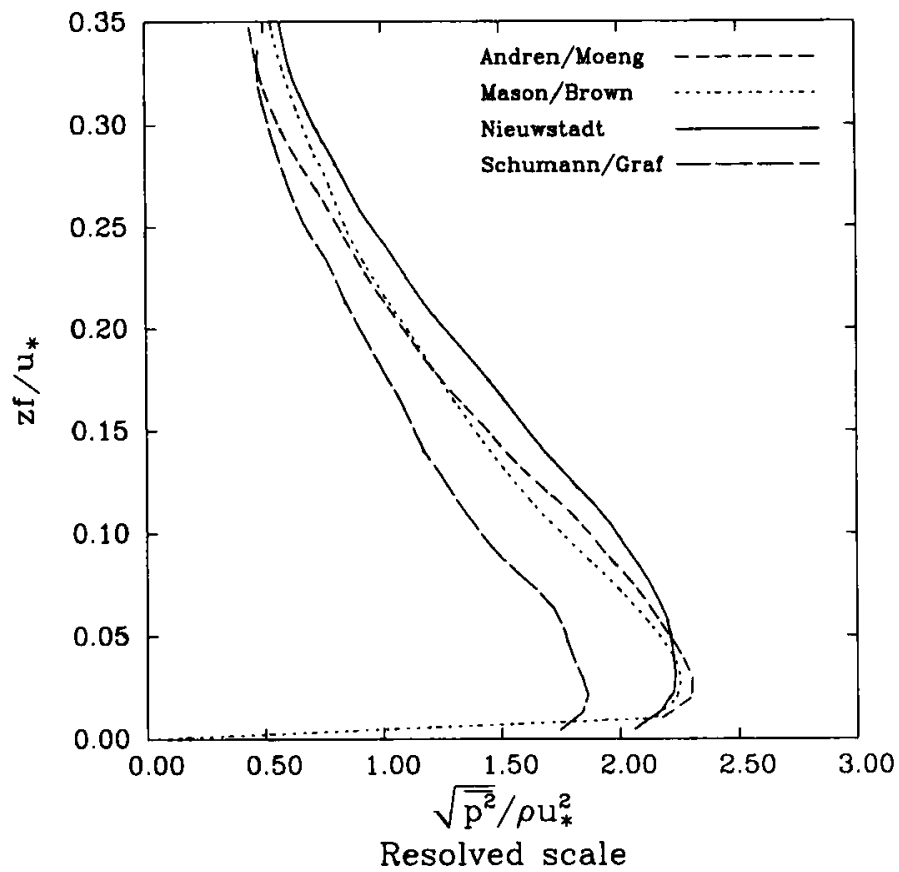

Figure 17. Normalized profiles of the standard deviation of pressure. Data from the test run with fixed profiles of SGS eddy diffusivities. The run by Mason-Brown is on a uniform vertical grid, without backscatter.

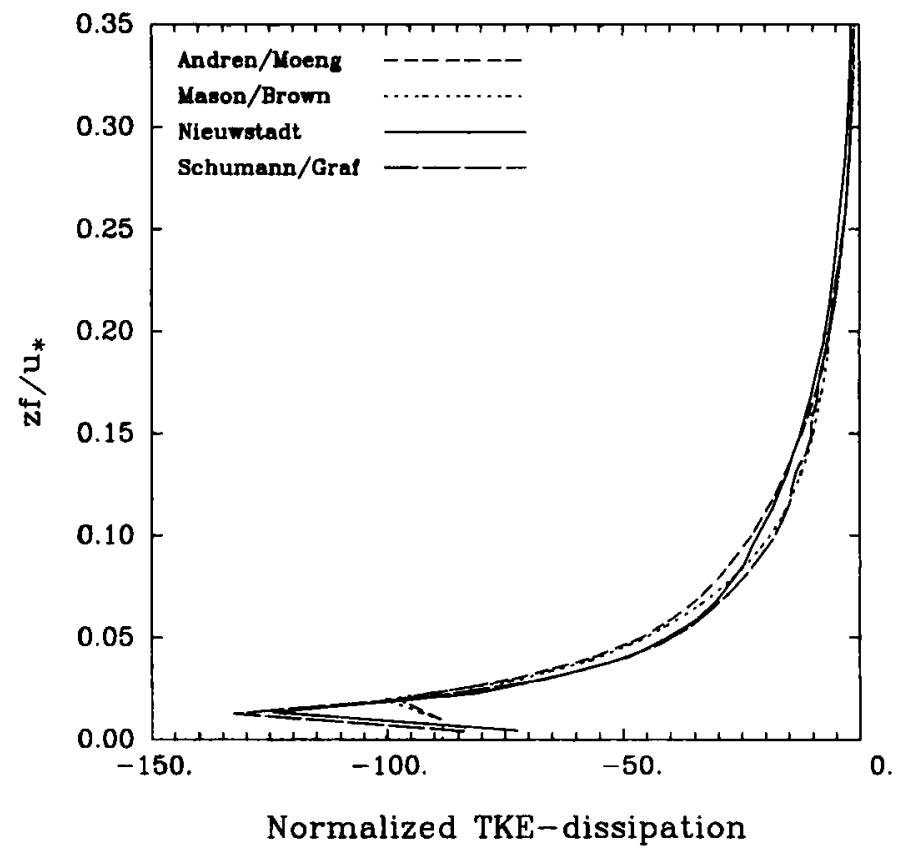

Figure 18. Normalized profiles of dissipation of resolved-scale TKE for the test run with fixed profiles of SGS eddy diffusivities. The run by Mason-Brown is on a uniform vertical grid, without backscatter. 
schemes used by Mason-Brown and Nieuwstadt are identical, and accordingly they should produce similar results (within the limits given by the rather short sampling period of the test). This is the case in the lower part of the boundary layer, as shown in Fig. 19. However, we do find some differences at higher levels (not shown) where Nieuwstadt's spectra are significantly flatter than Mason-Brown's, and also significantly flatter than in Nieuwstadt's run presented in section 4 . This is consistent with the smaller $K_{M}$ used in this test run. In the upper half of the boundary layer, we still find a steeper decrease in the runs by Mason-Brown and Schumann-Graf than in the runs by Andrén-Moeng and Nieuwstadt. For the latter two codes, the scalar exhibits a flat spectrum. In AndrénMoeng's results, there is also a tendency for energy to pile up at the highest wavenumbers, indicating that the prescribed SGS eddy diffusivities may be too small for the resolvedscale strain rates that result after dealiasing. We notice that Mason-Brown's spectra show slightly more energy at higher wavenumbers than their original non-backscatter
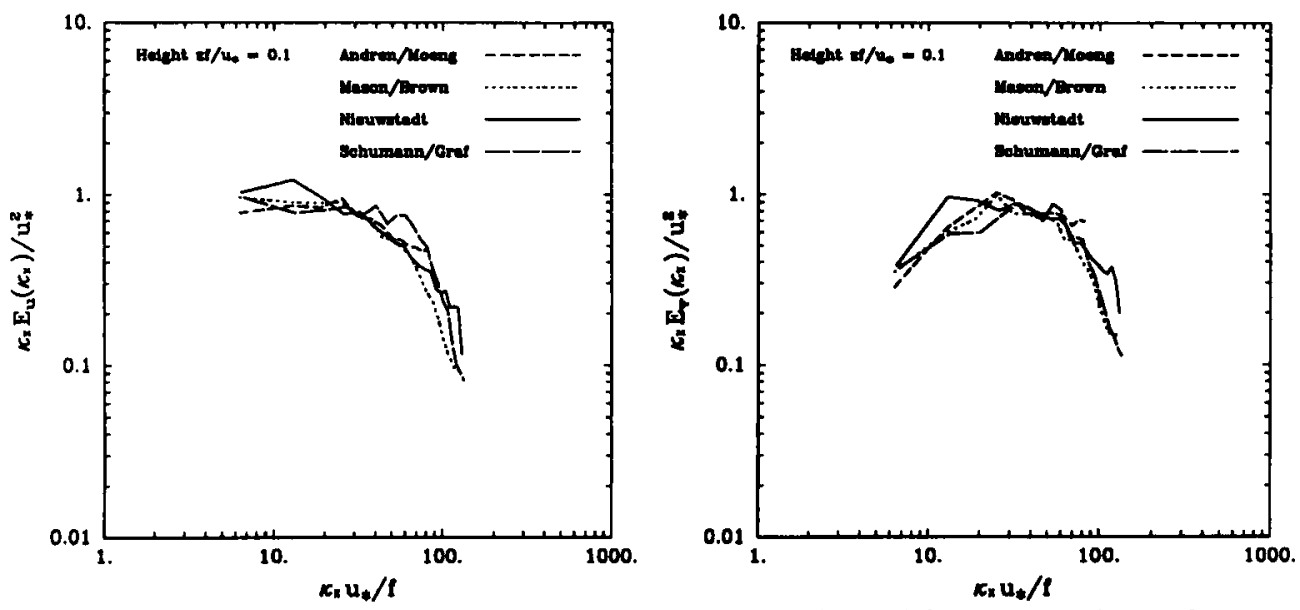

Horizontal energy spectrum of u

Horizontal energy spectrum of $v$
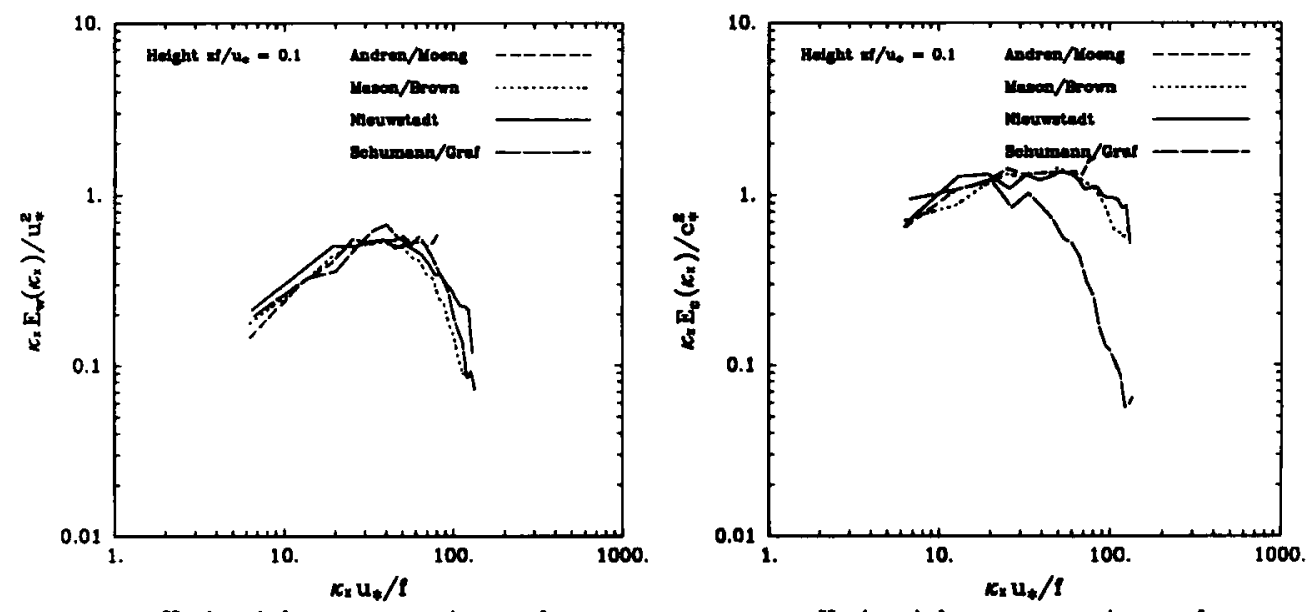

Horizontal energy spectrum of $\mathbf{w}$

Horizontal enerey spectrum of $c$

Figure 19. Spectra as in Fig. 15 but for the test run with fixed profiles of SGS eddy diffusivities. The run by Mason-Brown is on a uniform vertical grid, without backscatter. 
run. Schumann-Graf's spectra show a similar but smaller change. Their results for the passive scalar also indicate the diffusive property of the Smolarkiewicz advection scheme.

The convergence of spectral shapes at lower levels with differences still remaining at higher levels, where turbulence time-scales are larger, may be an indication that the test run is too short for all spectra to reach stationary conditions. Thus we do not view the remaining differences between the codes as indications of effects of different numerical solution schemes. Rather we take the general convergency of profiles, budgets, and, to a lesser extent, spectra as an indication that the numerics have a very little impact on the LES solutions, comparing with the SGS effect.

\section{Conclusions}

A shallow neutrally stratified PBL has been simulated using four different LES codes. The grid used is rather coarse and, as a result, spectra do not show a well defined inertial subrange. This implies that the Kolmogorov theory used to formulate the SGS models is poorly satisfied and we should expect to find results significantly influenced by SGS effects. In addition to the differences in SGS models and numerical schemes, Mason's code has also been run with a formulation that includes the stochastic backscatter of energy and scalar variance.

We have found that second-order moments as well as the budgets of such moments are sensitive to the magnitude of the SGS eddy diffusivities. In general we found that statistics of this neutrally stratified flow were more sensitive to the SGS model than corresponding statistics in the convective flow studied by NMMS92. Resolved-scale motions are generally more intense in the lower part of the boundary layer in the runs with smaller SGS eddy diffusivities. The spectra show that a decrease in SGS eddy diffusivities pushes the spectral peaks to higher wavenumbers. The inclusion of stochastic backscatter has a similar effect on the spectra and on the resolved-scale second moments and budgets. For the mean fields, our findings support the result of Mason and Thomson (1992) that the failure of the Smagorinsky SGS model in producing logarithmic mean gradients near the wall cannot be corrected by changing the value of the Smagorinsky coefficient. Differences between backscatter and non-backscatter runs are in general largest close to the surface, but also noticeable in the lower one-third of the boundary layer.

We have isolated the effect of the different numerical schemes by running a short test period with fixed profiles of SGS eddy diffusivities, which effectively eliminated the effect of the SGS models. Analysis of second-moment profiles, budgets, and spectra showed a clear convergence of results from the different LES codes. We conclude that the effect of the differences in numerical schemes is minor.

\section{APPENDIX}

As mentioned in section 1, all LESs were started from the same initial conditions. These initial fields were obtained from a PBL model using a single-point closure turbulence scheme tailored to give a rather shallow neutral PBL. Small random perturbations in all velocity components were added to this mean flow. The random perturbations were generated from random numbers uniformly distributed between -0.5 and 0.5 , and scaled to give energy $q^{2} / 2$. Initial profiles are given in Table A.1. 
TABLE A.1. INITIAL PROFILES USED FOR THE LES

\begin{tabular}{|c|c|c|c|}
\hline Height (m) & $\langle\bar{u}\rangle\left(\mathrm{m} \mathrm{s}^{-1}\right)$ & $\langle\bar{v}\rangle\left(\mathrm{m} \mathrm{s}^{-1}\right)$ & $\frac{q^{2}}{2}\left(m^{2} s^{-2}\right)$ \\
\hline 18.75 & 4.44 & 2.18 & 0.365 \\
\hline 56.25 & 5.92 & 2.67 & 0.295 \\
\hline 93.75 & 6.91 & 2.83 & 0.245 \\
\hline 131.25 & 7.73 & 2.84 & 0.205 \\
\hline 168.75 & 8.43 & 2.75 & 0.175 \\
\hline 206.25 & 9.02 & 2.57 & 0.145 \\
\hline 243.75 & 9.52 & 2.34 & 0.120 \\
\hline 281.25 & 9.93 & 2.06 & 0.100 \\
\hline 318.75 & 10.25 & 1.75 & 0.085 \\
\hline 356.25 & 10.47 & 1.44 & 0.070 \\
\hline 393.75 & 10.62 & 1.12 & 0.055 \\
\hline 431.25 & 10.70 & 0.82 & 0.045 \\
\hline 468.75 & 10.71 & 0.55 & 0.035 \\
\hline 506.25 & 10.67 & 0.31 & 0.025 \\
\hline 543.75 & 10.59 & 0.12 & 0.020 \\
\hline 581.25 & 10.48 & -0.02 & 0.015 \\
\hline 618.75 & 10.36 & -0.11 & 0.010 \\
\hline 656.25 & 10.24 & -0.16 & 0.010 \\
\hline 693.75 & 10.13 & -0.17 & 0.005 \\
\hline 731.25 & 10.04 & -0.15 & 0.005 \\
\hline 768.75 & 9.99 & -0.11 & 0.005 \\
\hline 806.25 & 9.96 & -0.06 & 0.000 \\
\hline 843.75 & 9.95 & -0.02 & 0.000 \\
\hline 881.25 & 9.96 & 0.01 & 0.000 \\
\hline 918.75 & 9.98 & 0.02 & 0.000 \\
\hline 956.25 & 9.99 & 0.02 & 0.000 \\
\hline 993.75 & 10.00 & 0.02 & 0.000 \\
\hline 1031.25 & 9.99 & 0.02 & 0.000 \\
\hline 1068.75 & 9.99 & 0.02 & 0.000 \\
\hline 1106.25 & 9.99 & 0.01 & 0.000 \\
\hline 1143.75 & 10.00 & 0.01 & 0.000 \\
\hline 1181.25 & 10.00 & 0.01 & 0.000 \\
\hline 1218.75 & 10.00 & 0.01 & 0.000 \\
\hline 1256.25 & 10.00 & 0.00 & 0.000 \\
\hline 1293.75 & 10.00 & 0.00 & 0.000 \\
\hline 1331.25 & 10.00 & 0.00 & 0.000 \\
\hline 1368.75 & 10.00 & 0.00 & 0.000 \\
\hline 1406.25 & 10.00 & 0.00 & 0.000 \\
\hline 1443.75 & 10.00 & 0.00 & 0.000 \\
\hline 1481.25 & 10.00 & 0.00 & 0.000 \\
\hline
\end{tabular}

Andrén, A. and Moeng, C.-H.

Lesieur, $M$.

Leslie, D. C. and Quarini, G. L.

Lilly, D. K.

Mansour, N. N., Moin, P., Reynolds, W. C. and Ferziger, J. H

Mason, P. J.

Mason, P. J. and Brown, A. R.

\section{REFERENCES}

1993 Single-point closures in a neutrally stratified boundary layer. J. Atmos. Sci., 50, 3366-3379

1990 Turbulence in fluids. Kluwer Academic Publishers, Dordrecht 1979 The application of turbulence theory to the formulation of subgrid modelling procedures. J. Fluid. Mech., 91, 65-91

1965

On the computational stability of numerical solutions of time dependent nonlinear geophysical fluid dynamics problems. Mon. Weather Rev., 93, 11-26

1979

'Improved methods for large eddy simulation of turbulence'. Pp. 386-401 in Turbulent shear flows 1. Eds. F. Durst et al. Springer Verlag, Berlin

1989 Large-eddy simulation of the convective boundary layer. $J$. Atmos. Sci. , 46, 1492-1516

1993

The sensitivity of large-eddy simulations of turbulent shear flow to subgrid models. Submitted to Boundary-Layer Meteorol. 
Mason, P. J. and Thomson, D. J.

Moeng, C.-H.

Moeng, C.-H. and Wyngaard, J. C. 1989

Nieuwstadt, F. T. M. and

Brost, R. A.

Nieuwstadt, F. T. M., Mason, P. J., 1992

Moeng, C.-H. and

Schumann, U.

Piacsek, S. A. and Williams, G. P. 1970

Schmidt, H. and Schumann, U. 1989

Schumann, U.

1975

Smagorinsky, J.

1963

Smolarkiewicz, P. K.
1992 Stochastic backscatter in large-eddy simulation of boundary layers. J. Fluid Mech., 242, 51-78

1984 A large-eddy-simulation model for the study of planetary boundary-layer turbulence. J. Atmos. Sci., 41, 2052-2062

Evaluation of turbulent transport and dissipation closures in second-order modeling. J. Atmos. Sci., 46, 2311-2330

1986 The decay of convective turbulence. J. Atmos. Sci., 43, 532546

'Large-eddy simulation of the convective boundary layer: A comparison of four computer codes'. Pp. 343-367 in Turbulent shear flows 8. Eds. F. Durst et al. Springer-Verlag, Berlin

Conservation properties of convection difference schemes. $J$. Comput. Phys., 6, 392-405

Coherent structure of the convective boundary layer derived from large-eddy simulation. J. Fluid Mech., 200, 511-562

Subgrid scale model for finite difference simulations of turbulent flows in plane channels and annuli. J. Comput. Phys., 18, 376-404

General circulation experiments with the primitive equations. Part 1: The basic experiment. Mon. Weather Rev., 91, 99-164

1984 A fully multidimensional positive definite advection transport algorithm with small implicit diffusion. J. Comput. Phys., 54, 325-362 